\title{
Antimicrobial susceptibility of 6685 organisms isolated from Canadian hospitals: CANWARD 2007
}

\author{
George G Zhanel PhD ${ }^{1,2,3}$, Mel DeCorby Msc ${ }^{1,3}$, Kim A Nichol MSc ${ }^{1,3}$, Aleksandra Wierzbowski MSc ${ }^{1,3}$, \\ Patricia J Baudry $\mathrm{MSc}^{1,3}$, Franil Tailor $\mathrm{BSc}^{1}$, Philippe Lagacé-Wiens $\mathrm{MD}^{1,2,3}$, Andrew Walkty $\mathrm{MD}^{1,2,3}$, \\ Sergio Fanella $M D^{1,2,3}$, Oscar Larios $M D^{1,2,3}$, Michael R Mulvey $\mathrm{PhD}^{1,4}$, Melissa McCracken MSc ${ }^{1,4}$, \\ James A Karlowsky PhD ${ }^{1,3}$, The Canadian Antimicrobial Resistance Alliance (CARA), Daryl J Hoban PhD ${ }^{1,3}$
}

GG Zhanel, M DeCorby, KA Nichol, et al. Antimicrobial susceptibility of 6685 organisms isolated from Canadian hospitals: CANWARD 2007. Can J Infect Dis Med Microbiol 2009;20(Suppl A):20A-30A

BACKGROUND: Antimicrobial resistance is a growing problem in North American hospitals as well as hospitals worldwide.

OBJECTIVES: To assess the antimicrobial susceptibility patterns of commonly used agents against the 20 most common organisms isolated from Canadian hospitals.

METHODS: In total, 7881 isolates were obtained between January 1, 2007, and December 31, 2007, from 12 hospitals across Canada as part of the Canadian Ward Surveillance Study (CANWARD 2007). Of these, 6685 isolates (20 most common organisms) obtained from bacteremic, urinary, respiratory and wound specimens underwent antimicrobial susceptibility testing. Susceptibility testing was assessed using the Clinical and Laboratory Standards Institute broth microdilution method.

RESULTS: The most active (based upon minimum inhibitory concentration [MIC] data only) agents against methicillin-resistant Staphylococcus aureus (MRSA) and methicillin-resistant Staphylococcus epidermidis (MRSE) were dalbavancin, daptomycin, linezolid, telavancin, tigecycline and vancomycin, with MICs required to inhibit the growth of $90 \%$ of organisms $\left(\mathrm{MIC}_{90}\right)$ of $0.06 \mu \mathrm{g} / \mathrm{mL}$ and $0.06 \mu \mathrm{g} / \mathrm{mL}$, $0.25 \mu \mathrm{g} / \mathrm{mL}$ and $0.25 \mu \mathrm{g} / \mathrm{mL}, 4 \mu \mathrm{g} / \mathrm{mL}$ and $1 \mu \mathrm{g} / \mathrm{mL}, 0.25 \mu \mathrm{g} / \mathrm{mL}$ and $0.25 \mu \mathrm{g} / \mathrm{mL}, 0.5 \mu \mathrm{g} / \mathrm{mL}$ and $0.25 \mu \mathrm{g} / \mathrm{mL}$, and $1 \mu \mathrm{g} / \mathrm{mL}$ and $2 \mu \mathrm{g} / \mathrm{mL}$, respectively. The most active agents against vancomycin-resistant enterococci were daptomycin, linezolid and tigecycline with $\mathrm{MIC}_{90} \mathrm{~S}$ of $2 \mu \mathrm{g} / \mathrm{mL}, 4 \mu \mathrm{g} / \mathrm{mL}$ and $0.12 \mu \mathrm{g} / \mathrm{mL}$, respectively. The most active agents against Escherichia coli were amikacin, cefepime, ertapenem, meropenem, piperacillin-tazobactam and tigecycline with $\mathrm{MIC}_{90}$ s of $4 \mu \mathrm{g} / \mathrm{mL}, 2 \mu \mathrm{g} / \mathrm{mL}, 0.06 \mu \mathrm{g} / \mathrm{mL}$ or less, $0.12 \mu \mathrm{g} / \mathrm{mL}$ or less, $4 \mu \mathrm{g} / \mathrm{mL}$ and $1 \mu \mathrm{g} / \mathrm{mL}$, respectively. The most active agents against extendedspectrum beta-lactamase-producing $E$ coli were ertapenem, meropenem and tigecycline with $\mathrm{MIC}_{90} \mathrm{~s}$ of $0.12 \mu \mathrm{g} / \mathrm{mL}$ or less, $0.12 \mu \mathrm{g} / \mathrm{mL}$ or less and $1 \mu \mathrm{g} / \mathrm{mL}$, respectively. The most active agents against Pseudomonas aeruginosa were amikacin, cefepime, meropenem and piperacillin-tazobactam with $\mathrm{MIC}_{90} \mathrm{~s}$ of $32 \mu \mathrm{g} / \mathrm{mL}, 32 \mu \mathrm{g} / \mathrm{mL}, 8 \mu \mathrm{g} / \mathrm{mL}$ and $64 \mathrm{\mu g} / \mathrm{mL}$, respectively. The most active agents against Stenotrophomonas maltophilia were tigecycline and trimethoprimsulfamethoxazole and levofloxacin with $\mathrm{MIC}_{90}$ s of $8 \mu \mathrm{g} / \mathrm{mL}, 8 \mu \mathrm{g} / \mathrm{mL}$ and $8 \mu \mathrm{g} / \mathrm{mL}$, respectively. The most active agents against Acinetobacter baumannii were amikacin, fluoroquinolones (eg, levofloxacin), meropenem, and tigecycline with $\mathrm{MIC}_{90} \mathrm{~s}$ of $2 \mu \mathrm{g} / \mathrm{mL}$ or less, $1 \mu \mathrm{g} / \mathrm{mL}, 4 \mu \mathrm{g} / \mathrm{mL}$ and $2 \mu \mathrm{g} / \mathrm{mL}$, respectively.
CONCLUSIONS: The most active agents versus Gram-positive cocci from Canadian hospitals were vancomycin, linezolid, daptomycin, tigecycline, dalbavancin and telavancin. The most active agents versus Gram-negative bacilli from Canadian hospitals were amikacin, cefepime, ertapenem (not $P$ aeruginosa), meropenem, piperacillintazobactam and tigecycline (not $P$ aeruginosa). Colistin (polymyxin E) was very active against $P$ aeruginosa and $A$ baumannii.

Key Words: Canadian hospitals; Resistance; Susceptibility

\section{La susceptibilité aux antimicrobiens de 6685 organismes isolés dans des hôpitaux canadiens : CANWARD 2007}

\begin{abstract}
HISTORIQUE : La résistance aux antimicrobiens est un problème croissant dans les hôpitaux nord-américains et du monde entier.

OBJECTIFS : Évaluer les modes de susceptibilité aux antimicrobiens d'agents souvent utilisés contre les 20 principaux organismes isolés dans des hôpitaux canadiens.

MÉTHODOLOGIE : Au total, on a recueilli 7881 isolats entre le $1^{\text {er }}$ janvier et le 31 décembre 2007 dans 12 hôpitaux du Canada, dans le cadre de l'étude CANWARD 2007 sur la surveillance des services aux hospitalisés canadiens. De ce nombre, 6685 isolats (les 20 principaux organismes) prélevés dans des échantillons bactériémiques, urinaires, respiratoires et de plaies ont subi un test de susceptibilité aux antimicrobiens. On a évalué ce test au moyen de la méthode de microdilution en milieu liquide du Clinical and Laboratory Standards Institute.

RÉSULTATS : Les agents les plus actifs (d'après les données de concentration minimale inhibitrice [CMI] seulement) contre le staphylocoque doré méthicillinorésistant (SARM) et le Staphylococcus epidermidis méthicillinorésistant (SERM) étaient la dalbavancine, la daptomycine, le linézolide, la télavancine, la tigécycline et la vancomycine, les CMI nécessaires pour inhiber la croissance de $90 \%$ des organismes $\left(\mathrm{CMI}_{90}\right)$ étant de $0,06 \mu \mathrm{g} / \mathrm{mL}$ et $0,06 \mu \mathrm{g} / \mathrm{mL}, 0,25 \mu \mathrm{g} / \mathrm{mL}$ et $0,25 \mu \mathrm{g} / \mathrm{mL}$, $4 \mu \mathrm{g} / \mathrm{mL}$ et $1 \mu \mathrm{g} / \mathrm{mL}, 0,25 \mu \mathrm{g} / \mathrm{mL}$ et $0,25 \mu \mathrm{g} / \mathrm{mL}, 0,05 \mu \mathrm{g} / \mathrm{mL}$ et $0,25 \mu \mathrm{g} / \mathrm{mL}$ et $1 \mu \mathrm{g} / \mathrm{mL}$ et $2 \mu \mathrm{g} / \mathrm{mL}$, respectivement. Les agents les plus actifs contre les entérocoques résistant à la vancomycine étaient la daptomycine, le linézolide et la tigécycline, avec une $\mathrm{CMI}_{90}$ de $2 \mu \mathrm{g} / \mathrm{mL}, 4 \mu \mathrm{g} / \mathrm{mL}$ et $0,12 \mu \mathrm{g} / \mathrm{mL}$, respectivement. Les agents les plus actifs contre l'Escherichia coli étaient l'amikacine, le céfépime, l'ertapénem, le méropénem, la pipéracilline-tazobactam et la tigécycline, avec une $\mathrm{CMI}_{90}$ de $4 \mu \mathrm{g} / \mathrm{mL}$, $2 \mu \mathrm{g} / \mathrm{mL}, 0,06 \mu \mathrm{g} / \mathrm{mL}$ ou moins, $0,12 \mu \mathrm{g} / \mathrm{mL}$ ou moins, $4 \mu \mathrm{g} / \mathrm{mL}$ et $1 \mu \mathrm{g} / \mathrm{mL}$, respectivement. Les agents les plus actifs contre l'E coli producteur de
\end{abstract}

${ }^{1}$ Department of Medical Microbiology, Faculty of Medicine, University of Manitoba; ${ }^{2}$ Departments of Medicine; ${ }^{3}$ Clinical Microbiology, Health Sciences Centre, MS673-Microbiology; ${ }^{4}$ Nosocomial Infections Branch, National Microbiology Laboratory, Health Canada, Winnipeg, Manitoba

Correspondence: Dr GG Zhanel, Clinical Microbiology, Health Sciences Centre, MS673-820 Sherbrook Street, Winnipeg, Manitoba R3A 1 R9.

Telephone 204-787-4902, fax 204-787-4699, e-mail ggzhanel@pcs.mb.ca 
bêta-lactamase à large spectre étaient l'ertapénem, le méropénem et la tigécycline, avec une $\mathrm{CMI}_{90}$ de $0,12 \mu \mathrm{g} / \mathrm{mL}$ ou moins, $0,12 \mu \mathrm{g} / \mathrm{mL}$ ou moins et $1 \mu \mathrm{g} / \mathrm{mL}$, respectivement. Les agents les plus actifs contre le Pseudomonas aeruginosa étaient l'amikacine, le céfépime, le méropénem et la pipéracillinetazobactam, avec une $\mathrm{CMI}_{90}$ de $32 \mu \mathrm{g} / \mathrm{mL}, 32 \mu \mathrm{g} / \mathrm{mL}, 8 \mu \mathrm{g} / \mathrm{mL}$ et $64 \mu \mathrm{g} / \mathrm{mL}$, respectivement. Les agents les plus actifs contre le Stenotrophomonas maltophilia étaient la tigécycline, le triméthoprim-sulfaméthoxazole et la lévoflocacine, avec une $\mathrm{CMI}_{90}$ de $8 \mu \mathrm{g} / \mathrm{mL}, 8 \mu \mathrm{g} / \mathrm{mL}$ et $8 \mu \mathrm{g} / \mathrm{mL}$, respectivement. Les agents les plus actifs contre l'Acinetobacter baumannii étaient l'amikacine, les fluoroquinolones (p. ex., la lévofloxacine), le méropénem et la tigécycline, avec une $\mathrm{CMI}_{90}$ de $2 \mu \mathrm{g} / \mathrm{mL}$ ou moins, $1 \mu \mathrm{g} / \mathrm{mL}$, $4 \mu \mathrm{g} / \mathrm{mL}$ et $2 \mu \mathrm{g} / \mathrm{mL}$, respectivement.

CONCLUSIONS : Les agents les plus actifs contre les cocci gram positifs des hôpitaux canadiens étaient la vancomycine, le linézolide, la daptomycine, la tigécycline, la dalbavancine et la télavancine. Les agents les plus actifs contre les bacilles gram négatifs des hôpitaux canadiens étaient l'amikacine, le céfépime, l'ertapénem (sauf pour le P aeruginosa), le méropénem, la pipéracilline-tazobactam et la tigécycline (sauf pour le $P$ aeruginosa). La colistine (polymyxine $E$ ) était très active contre le $P$ aeruginosa et l'A baumannii.
$\mathrm{H}$ ospitals in North America as well as hospitals worldwide are facing the growing presence of infections caused by antimicrobial-resistant as well as multidrug-resistant (MDR) pathogens (1-4). Pathogens including methicillin-resistantStaphylococcus aureus (MRSA; community-associated [CA-MRSA] and health care-associated [HA-MRSA]), vancomycin-resistant Enterococcus species (VRE), penicillin-resistant Streptococcus pneumoniae, extended-spectrum beta-lactamase (ESBL)-producing Escherichia coli and Klebsiella species, and fluoroquinolone-resistant and carbapenem-resistant Enterobacteriaceae and Pseudomonas aeruginosa are growing in prevalence in Canada, the United States and globally (5-10). Treatment options of antimicrobialresistant organisms can be severely limited because these organisms frequently display a MDR phenotype $(3,4)$.

We recently reported on the antimicrobial activity of commonly used agents against 3931 organisms isolated from intensive care units in Canada (11). The purpose of the present study was to assess the in vitro activity (minimum inhibitory concentrations required to inhibit the growth of $50 \%$ and $90 \%$ of organisms $\left[\mathrm{MIC}_{50}\right.$ and $\left.\mathrm{MIC}_{90}\right]$ ) of commonly prescribed antimicrobials against the 20 most common organisms (6685 isolates) obtained from patients in hospitals across Canada.

\section{METHODS}

\section{Bacterial isolates}

Study isolates were obtained as part of the Canadian Ward Surveillance Study (CANWARD 2007). The CANWARD study included 12 medical centres from all regions of Canada (www.can-r.ca). The precise methods of isolate collection are explained in detail in the first paper of the present supplement (12). In brief, from January 1, 2007, to December 31, 2007, inclusive, each centre collected and submitted clinical isolates from patients attending hospital clinics, emergency rooms, medical and surgical wards, and intensive care units. Each centre was asked to submit clinical isolates (consecutive, one organism per infection site per patient) from blood (360 isolates collected as 30 consecutive/month for each of the 12 months), respiratory $(\mathrm{n}=200)$, urine $(\mathrm{n}=100)$, and wound/ intravenous $(n=50)$ infections. All organisms were identified at the originating centre using local site criteria and were deemed clinically significant. In total, 7881 isolates were collected. Isolates were shipped to the reference laboratory (Health Sciences Centre, Winnipeg, Manitoba) on Amies charcoal swabs, subcultured onto appropriate media, and stocked in skim milk at $-80^{\circ} \mathrm{C}$ until MIC testing was carried out.

\section{Antimicrobial susceptibilities}

Susceptibility testing was carried out using microbroth dilution in accordance with the Clinical and Laboratory Standards Institute (CLSI) guidelines (11,13). For all antimicrobials tested, MIC interpretive standards were defined according to
CLSI breakpoints (CLSI 2006). Susceptibility testing could not be performed with all agents due to lack of space on the susceptibility panels. Thus, susceptibility testing was not performed with $P$ aeruginosa for ceftazidime, tobramycin and imipenem. The following interpretive breakpoints (Food and Drug Administration, USA) were used for tigecycline susceptible (S), intermediate (I) and resistant (R): $S$ aureus (methicillin-susceptible [MSSA] and MRSA) $0.5 \mu \mathrm{g} / \mathrm{mL}$ or less (S); Enterococcus faecalis (vancomycin susceptible), $0.25 \mu \mathrm{g} / \mathrm{mL}$ or less (S); Enterobacteriaceae, $2 \mu \mathrm{g} / \mathrm{mL}$ or less (S), $4 \mu \mathrm{g} / \mathrm{mL}$ (I), and $8 \mu \mathrm{g} / \mathrm{mL}$ or greater (R). No breakpoints are presently available for dalbavancin and telavancin.

\section{Characterization of MRSA, ESBL-producing \\ Enterobacteriaceae and VRE}

MRSA: Potential MRSA isolates were confirmed and tested as previously described (10). All isolates of MRSA were typed using pulsed-field gel electrophoresis following the Canadian standardized protocol to assess whether the isolates were CA-MRSA or HA-MRSA $(9,10,14,15)$.

ESBL testing: Potential E coli or Klebsiella species. ESBL producers were identified and tested as previously described (10).

VRE: Potential VRE isolates were confirmed using CLSI vancomycin disk diffusion testing and underwent vanA and van $\mathrm{B}$ polymerase chain reaction as well as DNA fingerprinting to assess genetic similarity, as previously described $(7,10)$.

\section{RESULTS}

Patient demographics and specimen types

A total of 7881 organisms (the 20 most common organisms, representing 6685 isolates, underwent susceptibility testing) were obtained from bacteremic, urinary, respiratory and wound specimens from hospitals across Canada. The patient demographics associated with these isolates have been described (12).

Most common organisms isolated from Canadian hospitals The 20 most common organisms isolated from hospitals across Canada included 3178 Gram-positive cocci: MSSA, S pneumoniae, MRSA, coagulase-negative staphylococci/ Staphylococcus epidermidis, and Enterococcus species, as well as 3507 Gram-negative bacilli including E coli, P aeruginosa, Klebsiella pneumoniae, Haemophilus influenzae, Enterobacter cloacae and Proteus mirabilis (12).

Antimicrobial activity against the 20 most common organisms isolated from Canadian hospitals (Gram-positive cocci) In vitro activity of various antimicrobials against MSSA, MRSA (including HA-MRSA and CA-MRSA), coagulase-negative 
staphylococci/S epidermidis (including both methicillin-susceptible [MSSE] and methicillin-resistant [MRSE] S epidermidis), S pneumoniae, Streptococcus pyogenes, Streptococcus agalactiae, Enterococcus faecalis and E faecium including VRE is displayed in Table 1. Limited resistance was observed against MSSA with the exception of clarithromycin (26.2\%), fluoroquinolones (range $9.5 \%$ to $12.0 \%$ ) and clindamycin (8.6\%) (Table 1). One hundred per cent susceptibility was observed with cefazolin, daptomycin, ertapenem, linezolid, meropenem, piperacillin-tazobactam, tigecycline and vancomycin. Dalbavancin and telavancin were active with $\mathrm{MIC}_{90} \mathrm{~s}$ of $0.06 \mu \mathrm{g} / \mathrm{mL}$ and $0.5 \mu \mathrm{g} / \mathrm{mL}$, respectively. Resistance rates with MRSA were $87.9 \%$ to $89.0 \%$ to fluoroquinolones, $90.5 \%$ to clarithromycin, $61.2 \%$ to clindamycin and $12.3 \%$ to trimethoprim-sulfamethoxazole (TMP-SMX). The most active agents tested against MRSA were vancomycin, daptomycin, linezolid and tigecycline with $100 \%$ susceptibility and $\mathrm{MIC}_{90} \mathrm{~s}$ of $1 \mu \mathrm{g} / \mathrm{mL}$, $0.25 \mu \mathrm{g} / \mathrm{mL}, 4 \mu \mathrm{g} / \mathrm{mL}$ and $0.5 \mu \mathrm{g} / \mathrm{mL}$, respectively (Table 1). Dalbavancin and telavancin were active against MRSA with $\mathrm{MIC}_{90} \mathrm{~s}$ of $0.06 \mu \mathrm{g} / \mathrm{mL}$ and $0.25 \mu \mathrm{g} / \mathrm{mL}$, respectively. Betalactams, ertapenem, meropenem, fluoroquinolones, clindamycin, clarithromycin and TMP-SMX were more active versus CA-MRSA than HA-MRSA (Table 1). The activity of dalbavancin, daptomycin, linezolid, telavancin, tigecycline and vancomycin did not change between HA-MRSA and CA-MRSA. Against MSSE, resistance was observed with clarithromycin at $64.8 \%$, clindamycin $38.9 \%$, fluoroquinolones $43.5 \%$ to $52.8 \%$ and TMP-SMX $41.7 \%$ (Table 1). One hundred per cent susceptibility was observed with daptomycin, linezolid and vancomycin. Dalbavancin and telavancin were active against MSSE with $\mathrm{MIC}_{90}$ of $0.06 \mu \mathrm{g} / \mathrm{mL}$ and $0.25 \mu \mathrm{g} / \mathrm{mL}$, respectively. The most active agents tested against MRSE were vancomycin, daptomycin and linezolid with 100\% susceptibility and $\mathrm{MIC}_{90} \mathrm{~s}$ of $2 \mu \mathrm{g} / \mathrm{mL}, 0.25 \mu \mathrm{g} / \mathrm{mL}$ and $1 \mu \mathrm{g} / \mathrm{mL}$, respectively (Table 1). Dalbavancin, tigecycline and telavancin were active against MRSE with $\mathrm{MIC}_{90} \mathrm{~s}$ of $0.06 \mu \mathrm{g} / \mathrm{mL}, 0.25 \mu \mathrm{g} / \mathrm{mL}$ and $0.25 \mu \mathrm{g} / \mathrm{mL}$, respectively.

With S pneumoniae, limited resistance was observed with the exception of clarithromycin at $13.0 \%$, clindamycin at $5.8 \%$, doxycycline at $4.4 \%$, fluoroquinolones (range $0.6 \%$ to 4.4\%) and TMP-SMX at 7.1\% (Table 1). One hundred per cent susceptibility was observed with linezolid and vancomycin with $\mathrm{MIC}_{90}$ s of $1 \mu \mathrm{g} / \mathrm{mL}$ and $0.25 \mu \mathrm{g} / \mathrm{mL}$ or less, respectively (Table 1 ). Dalbavancin, tigecycline and telavancin were active against $S$ pneumoniae with $\mathrm{MIC}_{90}$ of $0.03 \mu \mathrm{g} / \mathrm{mL}$ or less, $0.03 \mu \mathrm{g} / \mathrm{mL}$ or less and $0.06 \mu \mathrm{g} / \mathrm{mL}$ or less, respectively. Against $E$ faecalis, ciprofloxacin and levofloxacin resistance was $35.1 \%$ and $31.8 \%$, respectively. All E faecalis were susceptible to daptomycin, tigecycline and vancomycin. Dalbavancin and telavancin were active against $E$. faecalis with $\mathrm{MIC}_{90} \mathrm{~s}$ of $1 \mu \mathrm{g} / \mathrm{mL}$ and $1 \mu \mathrm{g} / \mathrm{mL}$, respectively. Against E faecium, ciprofloxacin and levofloxacin resistance was $82.8 \%$ and $79.3 \%$, respectively, while vancomycin resistance was $3.3 \%$. All E faecium were susceptible to daptomycin and tigecycline (Table 1). Dalbavancin and telavancin were active against $E$ faecium with $\mathrm{MIC}_{90} \mathrm{~s}$ of $0.25 \mu \mathrm{g} / \mathrm{mL}$ and $0.5 \mu \mathrm{g} / \mathrm{mL}$, respectively. The most active agents tested against VRE were daptomycin, linezolid and tigecycline with $\mathrm{MIC}_{90} \mathrm{~s}$ of $2 \mu \mathrm{g} / \mathrm{mL}, 4 \mu \mathrm{g} / \mathrm{mL}$ and $0.12 \mu \mathrm{g} / \mathrm{mL}$, respectively. Dalbavancin and telavancin demonstrated limited
TABLE 1

Antimicrobial activity against the $\mathbf{2 0}$ most common organisms isolated from Canadian hospitals (Grampositive cocci)

\begin{tabular}{|c|c|c|c|c|c|c|c|}
\hline & $\% \mathrm{~S}$ & $\%$ I & $\% \mathbf{R}$ & $\mathrm{MIC}_{50}$ & $\mathrm{MIC}_{90}$ & $\begin{array}{l}\text { Range } \\
\text { Min }\end{array}$ & $\begin{array}{l}\text { Range } \\
\text { Max }\end{array}$ \\
\hline \multicolumn{8}{|c|}{ Methicillin-susceptible Staphylococcus aureus ( $n=1095)$} \\
\hline Cefazolin & 100 & & & $\leq 0.5$ & 1 & $\leq 0.5$ & 2 \\
\hline Cefepime & 99.8 & 0.2 & & 4 & 8 & $\leq 1$ & 16 \\
\hline Ceftriaxone & 99.6 & 0.4 & & 4 & 4 & 1 & 16 \\
\hline Ciprofloxacin & 83.7 & 4.2 & 12 & 0.5 & 8 & $\leq 0.06$ & $>16$ \\
\hline Clarithromycin & 73.2 & 0.6 & 26.2 & $\leq 0.25$ & $>16$ & $\leq 0.03$ & $>32$ \\
\hline Clindamycin & 91 & 0.4 & 8.6 & $\leq 0.25$ & 0.25 & $\leq 0.12$ & $>8$ \\
\hline Dalbavancin & No BP & & & 0.06 & 0.06 & $\leq 0.03$ & 0.25 \\
\hline Daptomycin & 100 & & & 0.12 & 0.25 & $\leq 0.06$ & 1 \\
\hline Ertapenem & 100 & & & 0.25 & 0.25 & 0.12 & 0.5 \\
\hline Levofloxacin & 89.7 & 0.3 & 10 & 0.25 & 4 & $\leq 0.06$ & $>32$ \\
\hline Linezolid & 100 & & & 2 & 4 & $\leq 0.12$ & 4 \\
\hline Meropenem & 100 & & & $\leq 0.12$ & 0.12 & $\leq 0.06$ & 1 \\
\hline Moxifloxacin & 90 & 0.6 & 9.5 & $\leq 0.06$ & 1 & $\leq 0.06$ & $>16$ \\
\hline Nitrofurantoin & 100 & & & 16 & 16 & $\leq 0.5$ & 32 \\
\hline $\begin{array}{l}\text { Piperacillin/ } \\
\text { Tazobactam }\end{array}$ & 100 & & & $\leq 1$ & $\leq 1$ & $\leq 1$ & 8 \\
\hline Telavancin & No BP & & & 0.25 & 0.5 & $\leq 0.06$ & 1 \\
\hline Tigecycline & 100 & & & 0.25 & 0.25 & $\leq 0.03$ & 0.5 \\
\hline TMP/SMX & 99.3 & & 0.7 & $\leq 0.12$ & $\leq 0.12$ & $\leq 0.12$ & 16 \\
\hline Vancomycin & 100 & & & 1 & 1 & $\leq 0.25$ & 2 \\
\hline \multicolumn{8}{|c|}{ Methicillin-resistant $S$ aureus (MRSA) $(n=385)$} \\
\hline Cefazolin & & & $100.0^{*}$ & 64 & $>128$ & 0.5 & $>128$ \\
\hline Cefepime & & & $100.0^{*}$ & $>32$ & $>128$ & 2 & $>256$ \\
\hline Ceftriaxone & & & $100.0^{*}$ & $>64$ & $>256$ & 2 & $>256$ \\
\hline Ciprofloxacin & 10.8 & 0.2 & 89 & $>16$ & $>16$ & 0.25 & $>16$ \\
\hline Clindamycin & 38.6 & 0.3 & 61.2 & $>8$ & $>8$ & $\leq 0.12$ & $>8$ \\
\hline Clarithromycin & 9.5 & & 90.5 & $>16$ & $>32$ & $\leq 0.12$ & $>32$ \\
\hline Dalbavancin & No BP & & & 0.06 & 0.06 & $\leq 0.03$ & 0.12 \\
\hline Daptomycin & 100 & & & 0.12 & 0.25 & 0.12 & 1 \\
\hline Ertapenem & & & $100.0^{*}$ & 8 & $>32$ & 0.12 & $>32$ \\
\hline Levofloxacin & 11.6 & & 88.4 & $>32$ & $>32$ & 0.12 & $>32$ \\
\hline Linezolid & 100 & & & 2 & 4 & 0.25 & 4 \\
\hline Meropenem & & & $100.0^{*}$ & 8 & $>32$ & 0.12 & $>64$ \\
\hline Moxifloxacin & 11.6 & 0.5 & 87.9 & 8 & $>16$ & $\leq 0.06$ & $>16$ \\
\hline Nitrofurantoin & 100 & & & 16 & 16 & 8 & 32 \\
\hline $\begin{array}{l}\text { Piperacillin/ } \\
\text { Tazobactam }\end{array}$ & & & $100.0^{*}$ & 32 & 128 & $\leq 1$ & 256 \\
\hline Telavancin & No $B P$ & & & 0.25 & 0.25 & 0.12 & 1 \\
\hline Tigecycline & 100 & & & 0.25 & 0.5 & $\leq 0.03$ & 0.5 \\
\hline TMP/SMX & 87.7 & & 12.3 & $\leq 0.12$ & 8 & $\leq 0.12$ & $>8$ \\
\hline Vancomycin & 100 & & & 1 & 1 & $\leq 0.25$ & 2 \\
\hline \multicolumn{8}{|c|}{ Health care-associated MRSA $(n=285)$} \\
\hline Cefazolin & & & $100.0^{*}$ & 128 & $>128$ & 1 & $>128$ \\
\hline Cefepime & & & $100.0^{*}$ & 256 & $>256$ & 4 & $>32$ \\
\hline Ceftriaxone & & & $100.0^{*}$ & $>256$ & $>256$ & 2 & $>64$ \\
\hline Ciprofloxacin & 2.1 & & 97.9 & $>16$ & $>16$ & 0.25 & $>16$ \\
\hline Clindamycin & 25.3 & 0.3 & 74.4 & $>8$ & $>8$ & $\leq 0.25$ & $>8$ \\
\hline Clarithromycin & 3.2 & & 96.8 & $>16$ & $>16$ & $\leq 0.25$ & $>16$ \\
\hline Dalbavancin & No BP & & & 0.06 & 0.06 & $\leq 0.03$ & 0.12 \\
\hline Daptomycin & 100 & & & 0.12 & 0.25 & 0.12 & 1 \\
\hline Ertapenem & & & $100.0^{*}$ & 16 & $>32$ & 0.5 & $>32$ \\
\hline Levofloxacin & 2.1 & & 97.9 & $>32$ & $>32$ & 0.12 & $>32$ \\
\hline Linezolid & 100 & & & 2 & 4 & 0.25 & 4 \\
\hline Meropenem & & & $100.0^{*}$ & 8 & $>32$ & 0.25 & $>32$ \\
\hline
\end{tabular}


TABLE 1 - CONTINUED

Antimicrobial activity against the $\mathbf{2 0}$ most common organisms isolated from Canadian hospitals (Grampositive cocci)

\begin{tabular}{|c|c|c|c|c|c|c|c|}
\hline & $\% \mathrm{~S}$ & $\%$ I & $\% \mathbf{R}$ & $\mathrm{MIC}_{50}$ & $\mathrm{MIC}_{90}$ & $\begin{array}{l}\text { Range } \\
\text { Min }\end{array}$ & $\begin{array}{l}\text { Range } \\
\text { Max }\end{array}$ \\
\hline \multicolumn{8}{|c|}{ Health care-associated MRSA $(n=285)$ - CONTINUED } \\
\hline Moxifloxacin & 2.1 & 0.4 & 97.5 & 8 & $>16$ & $\leq 0.06$ & $>16$ \\
\hline Nitrofurantoin & 100 & & & 16 & 16 & 8 & 32 \\
\hline $\begin{array}{l}\text { Piperacillin/ } \\
\text { Tazobactam }\end{array}$ & & & $100.0^{*}$ & 64 & 128 & 2 & 256 \\
\hline Telavancin & No $B P$ & & & 0.25 & 0.25 & 0.12 & 1 \\
\hline Tigecycline & 100 & & & 0.25 & 0.5 & 0.12 & 0.5 \\
\hline TMP/SMX & 83.9 & & 16.1 & $\leq 0.12$ & $>8$ & $\leq 0.12$ & $>8$ \\
\hline Vancomycin & 100 & & & 1 & 1 & $\leq 0.25$ & 2 \\
\hline \multicolumn{8}{|c|}{ Community-associated MRSA $(n=71)$} \\
\hline Cefazolin & & & $100.0^{*}$ & 8 & 32 & 1 & 128 \\
\hline Cefepime & & & $100.0^{*}$ & 32 & $>32$ & 8 & $>32$ \\
\hline Ceftriaxone & & & $100.0^{*}$ & 32 & $>64$ & 16 & $>64$ \\
\hline Ciprofloxacin & 38 & 1.4 & 60.6 & 16 & $>16$ & 0.25 & $>16$ \\
\hline Clindamycin & 90.1 & & 9.9 & $\leq 0.25$ & $\leq 0.25$ & $\leq 0.25$ & $>8$ \\
\hline Clarithromycin & 28.2 & & 71.8 & $>16$ & $>16$ & $\leq 0.25$ & $>16$ \\
\hline Dalbavancin & No BP & & & 0.06 & 0.06 & $\leq 0.03$ & 0.12 \\
\hline Daptomycin & 100 & & & 0.12 & 0.5 & 0.12 & 0.5 \\
\hline Ertapenem & & & $100.0^{*}$ & 2 & 4 & 0.25 & 8 \\
\hline Levofloxacin & 42.3 & & 57.7 & 4 & 8 & 0.12 & 16 \\
\hline Linezolid & 100 & & & 2 & 2 & 1 & 4 \\
\hline Meropenem & & & $100.0^{*}$ & 1 & 4 & 0.25 & 8 \\
\hline Moxifloxacin & 42.3 & 1.4 & 56.3 & 2 & 2 & $\leq 0.06$ & 4 \\
\hline Nitrofurantoin & 100 & & & 16 & 16 & 16 & 16 \\
\hline $\begin{array}{l}\text { Piperacillin/ } \\
\text { Tazobactam }\end{array}$ & & & $100.0^{*}$ & 16 & 32 & 2 & 64 \\
\hline Telavancin & No BP & & & 0.25 & 0.25 & 0.12 & 0.5 \\
\hline Tigecycline & 100 & & & 0.25 & 0.25 & 0.06 & 0.25 \\
\hline TMP/SMX & 100 & & & $\leq 0.12$ & $\leq 0.12$ & $\leq 0.12$ & 1 \\
\hline Vancomycin & 100 & & & 1 & 1 & 0.5 & 1 \\
\hline \multicolumn{8}{|c|}{ Coagulase-negative staphylococci $(\mathrm{n}=182)$} \\
\hline Cefazolin & 84.8 & 1.1 & 14.1 & 1 & 64 & $\leq 0.5$ & $>128$ \\
\hline Cefepime & 71.7 & 9.8 & 18.5 & 4 & 128 & $\leq 1$ & $>128$ \\
\hline Ceftriaxone & 69.4 & 14.3 & 16.3 & 8 & $>256$ & 0.5 & $>256$ \\
\hline Ciprofloxacin & 38.8 & 1 & 60.2 & 16 & $>16$ & 0.12 & $>16$ \\
\hline Clarithromycin & 43.9 & 2 & 54.1 & 16 & $>16$ & 0.12 & $>32$ \\
\hline Clindamycin & 71.4 & & 28.6 & $\leq 0.25$ & $>8$ & $\leq 0.12$ & $>8$ \\
\hline Dalbavancin & No BP & & & $\leq 0.03$ & 0.06 & $\leq 0.03$ & 0.12 \\
\hline Daptomycin & 100 & & & 0.12 & 0.25 & $\leq 0.06$ & 0.5 \\
\hline Ertapenem & 83.3 & & 16.7 & 0.5 & $>4$ & 0.12 & $>4$ \\
\hline Levofloxacin & 39.8 & 4.1 & 56.1 & 8 & $>32$ & $\leq 0.06$ & $>32$ \\
\hline Linezolid & 100 & & & 1 & 1 & $\leq 0.12$ & 4 \\
\hline Meropenem & 75.5 & 9.2 & 15.3 & 1 & 16 & $\leq 0.06$ & 32 \\
\hline Moxifloxacin & 42.9 & 6.1 & 51 & 2 & $>16$ & $\leq 0.06$ & $>16$ \\
\hline $\begin{array}{l}\text { Piperacillin/ } \\
\text { Tazobactam }\end{array}$ & 88.8 & & 11.2 & $\leq 1$ & 16 & $\leq 1$ & 256 \\
\hline Telavancin & No BP & & & 0.12 & 0.12 & $\leq 0.06$ & 0.12 \\
\hline Tigecycline & No BP & & & 0.25 & 0.5 & 0.06 & 1 \\
\hline TMP/SMX & 64.3 & & 35.7 & 0.5 & 8 & $\leq 0.12$ & $>8$ \\
\hline Vancomycin & 100 & & & 1 & 2 & 0.5 & 2 \\
\hline \multicolumn{8}{|c|}{ Staphylococcus epidermidis $(n=135)$} \\
\hline Cefazolin & 83.1 & 1.5 & 15.4 & 1 & 64 & $\leq 0.5$ & 128 \\
\hline Cefepime & 72.3 & 6.9 & 20.8 & 4 & $>32$ & $\leq 0.25$ & 128 \\
\hline Ceftriaxone & 58.6 & 22.6 & 18.8 & 8 & $>64$ & $\leq 0.25$ & $>256$ \\
\hline Ciprofloxacin & 39.9 & & 60.1 & 8 & $>16$ & $\leq 0.06$ & $>16$ \\
\hline
\end{tabular}

TABLE 1 - CONTINUED

Antimicrobial activity against the 20 most common organisms isolated from Canadian hospitals (Grampositive cocci)

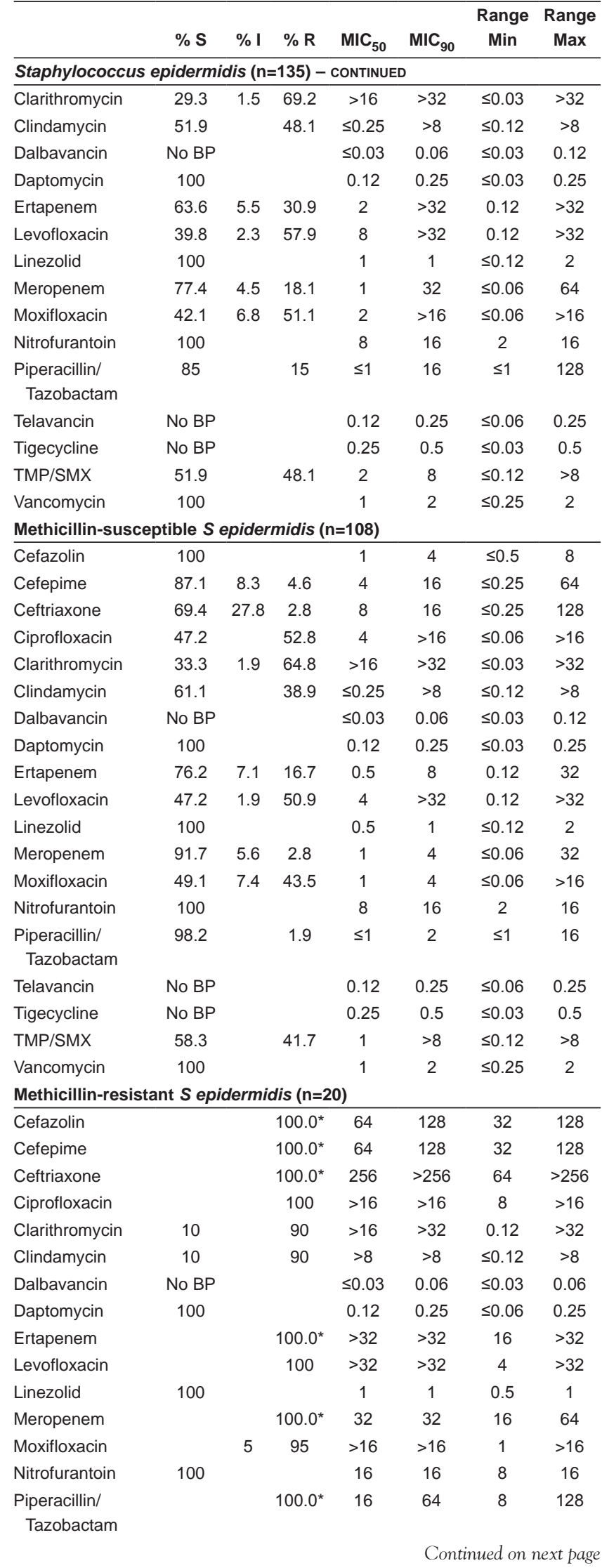


TABLE 1 - CONTINUED

Antimicrobial activity against the $\mathbf{2 0}$ most common organisms isolated from Canadian hospitals (Grampositive cocci)

\begin{tabular}{|c|c|c|c|c|c|c|c|}
\hline & $\% \mathrm{~S}$ & $\%$ I & $\% \mathrm{R}$ & $\mathrm{MIC}_{50}$ & $\mathrm{MIC}_{90}$ & $\begin{array}{l}\text { Range } \\
\text { Min }\end{array}$ & $\begin{array}{l}\text { Range } \\
\text { Max }\end{array}$ \\
\hline \multicolumn{8}{|c|}{ Methicillin-resistant $S$ epidermidis $(\mathrm{n}=\mathbf{2 0})$ - CONTINUED } \\
\hline Telavancin & No BP & & & 0.25 & 0.25 & 0.12 & 0.25 \\
\hline Tigecycline & No BP & & & 0.25 & 0.25 & 0.06 & 0.5 \\
\hline TMP/SMX & 25 & & 75 & 4 & 8 & $\leq 0.12$ & 8 \\
\hline Vancomycin & 100 & & & 1 & 2 & 1 & 2 \\
\hline \multicolumn{8}{|c|}{ Streptococcus pneumoniae - all $(n=702)$} \\
\hline $\begin{array}{l}\text { Amoxicillin/ } \\
\text { Clavulanate }\end{array}$ & 99.4 & 0.4 & 0.2 & $\leq 0.06$ & 0.12 & $\leq 0.06$ & 8 \\
\hline Cefuroxime & 95.4 & 2.1 & 2.5 & $\leq 0.25$ & $\leq 0.25$ & $\leq 0.25$ & $>16$ \\
\hline Ceftriaxone & 99.7 & 0.1 & 0.2 & $</ 0.06$ & 0.12 & $\leq 0.06$ & 4 \\
\hline Ciprofloxacin & 95.6 & & 4.4 & 1 & 2 & $\leq 0.06$ & $>16$ \\
\hline Clarithromycin & 80.9 & 6.1 & 13 & $\leq 0.03$ & 2 & $\leq 0.03$ & $>32$ \\
\hline Clindamycin & 94 & 0.2 & 5.8 & $\leq 0.12$ & $\leq 0.12$ & $\leq 0.12$ & $>8$ \\
\hline Dalbavancin & No BP & & & $\leq 0.03$ & $\leq 0.03$ & $\leq 0.03$ & 0.12 \\
\hline Daptomycin & No BP & & & $\leq 0.06$ & 0.12 & $\leq 0.06$ & 0.12 \\
\hline Doxycycline & 93 & 2.6 & 4.4 & $\leq 0.25$ & 1 & $\leq 0.25$ & $>16$ \\
\hline Ertapenem & 99.8 & & 0.2 & $\leq 0.06$ & $\leq 0.06$ & $\leq 0.06$ & 4 \\
\hline Levofloxacin & 99.4 & & 0.6 & 0.5 & 1 & $\leq 0.06$ & 32 \\
\hline Linezolid & 100 & & & 0.5 & 1 & $\leq 0.12$ & 2 \\
\hline Meropenem & 97.1 & 2.6 & 0.3 & $\leq 0.06$ & $\leq 0.06$ & $\leq 0.06$ & 2 \\
\hline Moxifloxacin & 99.1 & 0.3 & 0.6 & 0.12 & 0.25 & $\leq 0.06$ & 8 \\
\hline Penicillin & 79.1 & 15.7 & 5.2 & 0.06 & 0.25 & $\leq 0.03$ & 8 \\
\hline $\begin{array}{l}\text { Piperacillin/ } \\
\text { Tazobactam }\end{array}$ & No BP & & & $\leq 1$ & $\leq 1$ & $\leq 1$ & 4 \\
\hline Telavancin & No BP & & & $\leq 0.06$ & $\leq 0.06$ & $\leq 0.06$ & 0.12 \\
\hline Telithromycin & 100 & & & 0.015 & 0.3 & $\leq 0.008$ & 0.5 \\
\hline Tigecycline & No BP & & & $\leq 0.03$ & $\leq 0.03$ & $\leq 0.03$ & 0.12 \\
\hline TMP/SMX & 86.2 & 6.7 & 7.1 & $\leq 0.12$ & 1 & $\leq 0.12$ & $>8$ \\
\hline Vancomycin & 100 & & & $\leq 0.25$ & $\leq 0.25$ & $\leq 0.25$ & 0.5 \\
\hline \multicolumn{8}{|c|}{ Streptococcus pyogenes $(n=105)$} \\
\hline Ceftriaxone & 100 & & & $\leq 0.06$ & $\leq 0.06$ & $\leq 0.06$ & $\leq 0.06$ \\
\hline Ciprofloxacin & No BP & & & 1 & 2 & 0.25 & 4 \\
\hline Clarithromycin & 90.4 & & 9.6 & $\leq 0.03$ & 0.12 & $\leq 0.03$ & $>32$ \\
\hline Clindamycin & 97.3 & & 2.7 & $\leq 0.12$ & $\leq 0.12$ & $\leq 0.12$ & $>8$ \\
\hline Dalbavancin & No BP & & & $\leq 0.03$ & $\leq 0.03$ & $\leq 0.03$ & $\leq 0.03$ \\
\hline Daptomycin & 100 & & & $\leq 0.03$ & 0.06 & $\leq 0.03$ & 0.12 \\
\hline Ertapenem & 100 & & & $\leq 0.06$ & $\leq 0.06$ & $\leq 0.06$ & $\leq 0.06$ \\
\hline Levofloxacin & 98.6 & 1.4 & & 0.5 & 1 & 0.25 & 4 \\
\hline Linezolid & 100 & & & 1 & 1 & 0.5 & 2 \\
\hline Meropenem & 100 & & & $\leq 0.06$ & $\leq 0.06$ & $\leq 0.06$ & $\leq 0.06$ \\
\hline Moxifloxacin & No BP & & & 0.12 & 0.25 & $\leq 0.06$ & 0.5 \\
\hline $\begin{array}{l}\text { Piperacillin/ } \\
\text { Tazobactam }\end{array}$ & No BP & & & $\leq 1$ & $\leq 1$ & $\leq 1$ & $\leq 1$ \\
\hline Telavancin & No BP & & & $\leq 0.06$ & $\leq 0.06$ & $\leq 0.06$ & $\leq 0.06$ \\
\hline Tigecycline & 100 & & & $\leq 0.03$ & 0.06 & $\leq 0.03$ & 0.12 \\
\hline TMP/SMX & No BP & & & $\leq 0.12$ & $\leq 0.12$ & $\leq 0.12$ & 0.25 \\
\hline Vancomycin & 100 & & & 0.5 & 0.5 & $\leq 0.25$ & 0.5 \\
\hline \multicolumn{8}{|c|}{ Streptococcus agalactiae $(\mathrm{n}=116)$} \\
\hline Ceftriaxone & 100 & & & $\leq 0.06$ & $\leq 0.06$ & $\leq 0.06$ & 0.25 \\
\hline Ciprofloxacin & No BP & & & 1 & 2 & 0.5 & $>16$ \\
\hline Clarithromycin & 75 & 3.4 & 21.6 & $\leq 0.03$ & $>32$ & $\leq 0.03$ & $>32$ \\
\hline Clindamycin & 85.2 & 2.3 & 12.5 & $\leq 0.12$ & $>8$ & $\leq 0.12$ & $>8$ \\
\hline Dalbavancin & No BP & & & $\leq 0.03$ & $\leq 0.03$ & $\leq 0.03$ & $\leq 0.03$ \\
\hline Daptomycin & 100 & & & 0.12 & 0.12 & $\leq 0.03$ & 0.12 \\
\hline Ertapenem & 100 & & & $\leq 0.06$ & $\leq 0.06$ & $\leq 0.06$ & $\leq 0.06$ \\
\hline
\end{tabular}

TABLE 1 - CONTINUED

Antimicrobial activity against the 20 most common organisms isolated from Canadian hospitals (Grampositive cocci)

\begin{tabular}{|c|c|c|c|c|c|c|c|}
\hline & $\% \mathrm{~S}$ & $\%$ I & $\% \mathbf{R}$ & $\mathrm{MIC}_{50}$ & $\mathrm{MIC}_{90}$ & $\begin{array}{c}\text { Range } \\
\text { Min }\end{array}$ & $\begin{array}{c}\text { Range } \\
\text { Max }\end{array}$ \\
\hline \multicolumn{8}{|c|}{ Streptococcus agalactiae $(n=116)-$ CONTINUED } \\
\hline Levofloxacin & 97.7 & & 2.3 & 1 & 1 & 0.5 & $>32$ \\
\hline Linezolid & 100 & & & 1 & 1 & $\leq 0.12$ & 2 \\
\hline Meropenem & 100 & & & $\leq 0.06$ & $\leq 0.06$ & $\leq 0.06$ & $\leq 0.06$ \\
\hline Moxifloxacin & No BP & & & 0.12 & 0.25 & $\leq 0.06$ & 4 \\
\hline Nitrofurantoin & No data & & & & & & \\
\hline $\begin{array}{l}\text { Piperacillin/ } \\
\text { Tazobactam }\end{array}$ & No BP & & & $\leq 1$ & $\leq 1$ & $\leq 1$ & $\leq 1$ \\
\hline Telavancin & No BP & & & $\leq 0.06$ & $\leq 0.06$ & $\leq 0.06$ & $\leq 0.06$ \\
\hline Tigecycline & 100 & & & 0.06 & 0.12 & $\leq 0.03$ & 0.12 \\
\hline TMP/SMX & No BP & & & $\leq 0.12$ & $\leq 0.12$ & $\leq 0.12$ & 0.25 \\
\hline Vancomycin & 100 & & & 0.5 & 0.5 & $\leq 0.25$ & 0.5 \\
\hline \multicolumn{8}{|c|}{ Enterococcus, nonspeciated $(n=237)$} \\
\hline Cefazolin & No BP & & & 32 & 128 & $\leq 0.5$ & $>128$ \\
\hline Cefepime & No BP & & & 64 & $>128$ & $\leq 0.25$ & $>128$ \\
\hline Ceftriaxone & No BP & & & 256 & $>256$ & $\leq 0.25$ & $>256$ \\
\hline Ciprofloxacin & 31 & 24.6 & 44.4 & 2 & $>16$ & $\leq 0.06$ & $>16$ \\
\hline Clarithromycin & No BP & & & $>16$ & $>16$ & $\leq 0.03$ & $>32$ \\
\hline Clindamycin & No BP & & & $>8$ & $>8$ & $\leq 0.12$ & $>8$ \\
\hline Dalbavancin & No BP & & & 0.06 & 0.12 & $\leq 0.03$ & 0.5 \\
\hline Daptomycin & 100 & & & 0.5 & 1 & $\leq 0.03$ & 2 \\
\hline Ertapenem & No BP & & & 8 & $>32$ & $\leq 0.06$ & $>32$ \\
\hline Levofloxacin & 58.2 & 0.4 & 41.4 & 2 & $>32$ & $\leq 0.06$ & $>32$ \\
\hline Linezolid & 95.7 & 4.3 & & 2 & 2 & $\leq 0.12$ & 4 \\
\hline Meropenem & No BP & & & 8 & 16 & $\leq 0.06$ & $>64$ \\
\hline Moxifloxacin & No BP & & & 0.5 & $>16$ & $\leq 0.06$ & $>16$ \\
\hline Nitrofurantoin & 84 & 8 & 8 & 8 & 64 & $\leq 0.5$ & 128 \\
\hline $\begin{array}{l}\text { Piperacillin/ } \\
\text { Tazobactam }\end{array}$ & No BP & & & 4 & 16 & $\leq 1$ & $>512$ \\
\hline Telavancin & No BP & & & 0.5 & 1 & $\leq 0.06$ & 1 \\
\hline Tigecycline & No BP & & & 0.12 & 0.25 & $\leq 0.03$ & 1 \\
\hline Vancomycin & 99.1 & 0.9 & & 1 & 2 & $\leq 0.25$ & $>8$ \\
\hline \multicolumn{8}{|c|}{ Enterococcus faecalis $(n=161)$} \\
\hline Cefazolin & No BP & & & 32 & 128 & 0.5 & $>128$ \\
\hline Cefepime & No BP & & & $>32$ & 128 & $\leq 0.25$ & $>128$ \\
\hline Ceftriaxone & No BP & & & $>64$ & $>256$ & $\leq 0.25$ & $>256$ \\
\hline Ciprofloxacin & 38.3 & 26.6 & 35.1 & 2 & $>16$ & 0.25 & $>16$ \\
\hline Clarithromycin & No BP & & & 2 & $>32$ & 0.06 & $>32$ \\
\hline Clindamycin & No BP & & & $>8$ & $>8$ & $\leq 0.12$ & $>8$ \\
\hline Dalbavancin & No BP & & & 0.06 & 0.06 & $\leq 0.03$ & 0.25 \\
\hline Daptomycin & 100 & & & 0.5 & 1 & $\leq 0.06$ & 2 \\
\hline Ertapenem & No BP & & & 8 & 16 & 0.25 & $>32$ \\
\hline Levofloxacin & 68.2 & & 31.8 & 2 & $>32$ & 0.25 & $>32$ \\
\hline Linezolid & 98.7 & 1.3 & & 2 & 2 & 0.5 & 4 \\
\hline Meropenem & No BP & & & 4 & 8 & $\leq 0.06$ & $>32$ \\
\hline Moxifloxacin & No BP & & & 0.5 & 16 & $\leq 0.06$ & $>16$ \\
\hline Nitrofurantoin & 98.8 & 1.2 & & 8 & 16 & 2 & 64 \\
\hline $\begin{array}{l}\text { Piperacillin/ } \\
\text { Tazobactam }\end{array}$ & No BP & & & 4 & 8 & $\leq 1$ & 512 \\
\hline Telavancin & No BP & & & 0.5 & 1 & $\leq 0.06$ & 1 \\
\hline Tigecycline & 100 & & & 0.12 & 0.25 & 0.06 & 0.25 \\
\hline Vancomycin & 100 & & & 1 & 2 & 0.5 & 4 \\
\hline \multicolumn{8}{|c|}{ Enterococcus faecium $(n=60)$} \\
\hline Cefazolin & No BP & & & $>128$ & $>128$ & 32 & $>128$ \\
\hline Cefepime & No BP & & & $>32$ & $>128$ & 2 & $>128$ \\
\hline
\end{tabular}


TABLE 1 - CONTINUED

Antimicrobial activity against the $\mathbf{2 0}$ most common organisms isolated from Canadian hospitals (Grampositive cocci)

\begin{tabular}{|c|c|c|c|c|c|c|c|}
\hline & $\% \mathrm{~S}$ & $\%$ I & $\% \mathbf{R}$ & $\mathrm{MIC}_{50}$ & $\mathrm{MIC}_{90}$ & $\begin{array}{l}\text { Range } \\
\text { Min }\end{array}$ & $\begin{array}{l}\text { Range } \\
\text { Max }\end{array}$ \\
\hline \multicolumn{8}{|c|}{ Enterococcus faecium $(\mathrm{n}=60)$ - CONTINUED } \\
\hline Ceftriaxone & No BP & & & $>64$ & $>256$ & 0.5 & $>256$ \\
\hline Ciprofloxacin & 12.1 & 5.1 & 82.8 & $>16$ & $>16$ & 1 & $>16$ \\
\hline Clarithromycin & No BP & & & $>32$ & $>32$ & 0.5 & $>32$ \\
\hline Clindamycin & No BP & & & $>8$ & $>8$ & $\leq 0.12$ & $>8$ \\
\hline Dalbavancin & No BP & & & 0.12 & 0.25 & $\leq 0.03$ & $>16$ \\
\hline Daptomycin & 100 & & & 1 & 2 & 0.12 & 2 \\
\hline Ertapenem & No BP & & & $>32$ & $>32$ & 4 & $>32$ \\
\hline Levofloxacin & 17.2 & 3.5 & 79.3 & $>32$ & $>32$ & 1 & $>32$ \\
\hline Linezolid & 91.4 & 8.6 & & 2 & 2 & 1 & 4 \\
\hline Meropenem & No BP & & & $>32$ & $>64$ & 4 & $>64$ \\
\hline Moxifloxacin & No BP & & & $>16$ & $>16$ & $\leq 0.25$ & $>16$ \\
\hline Nitrofurantoin & 40.6 & 32.4 & 27 & 64 & 128 & 8 & 128 \\
\hline $\begin{array}{l}\text { Piperacillin/ } \\
\text { Tazobactam }\end{array}$ & No BP & & & 512 & $>512$ & 2 & $>512$ \\
\hline Telavancin & No BP & & & 0.12 & 0.5 & $\leq 0.06$ & 4 \\
\hline Tigecycline & 100 & & & 0.12 & 0.12 & 0.06 & 0.5 \\
\hline Vancomycin & 88.3 & & 11.7 & 0.5 & $>8$ & $\leq 0.25$ & $>8$ \\
\hline \multicolumn{8}{|c|}{ Vancomycin-resistant enterococci $(n=8)^{\dagger}$} \\
\hline Cefazolin & No BP & & & $>128$ & $>128$ & $>128$ & $>128$ \\
\hline Cefepime & No BP & & & $>128$ & $>128$ & $>32$ & $>128$ \\
\hline Ceftriaxone & No BP & & & $>256$ & $>256$ & $>64$ & $>256$ \\
\hline Ciprofloxacin & & & 100 & $>16$ & $>16$ & $>16$ & $>16$ \\
\hline Clarithromycin & No BP & & & $>16$ & $>32$ & 2 & $>32$ \\
\hline Clindamycin & No BP & & & $>8$ & $>8$ & $\leq 0.25$ & $>8$ \\
\hline Dalbavancin & No BP & & & 0.5 & $>16$ & 0.06 & $>16$ \\
\hline Daptomycin & 100 & & & 1 & 2 & 0.25 & 2 \\
\hline Ertapenem & No BP & & & $>32$ & $>32$ & $>32$ & $>32$ \\
\hline Levofloxacin & No BP & & 100 & $>32$ & $>32$ & $>32$ & $>32$ \\
\hline Linezolid & 75 & 25 & & 2 & 4 & 1 & 4 \\
\hline Meropenem & No BP & & & $>64$ & $>64$ & $>32$ & $>64$ \\
\hline Moxifloxacin & No BP & & & $>16$ & $>16$ & $>16$ & $>16$ \\
\hline Nitrofurantoin & & 50 & 50 & 64 & 128 & 64 & 128 \\
\hline $\begin{array}{l}\text { Piperacillin/ } \\
\text { Tazobactam }\end{array}$ & No BP & & & $>512$ & $>512$ & $>512$ & $>512$ \\
\hline Telavancin & No BP & & & 0.12 & 4 & 0.12 & 4 \\
\hline Tigecycline & No BP & & & 0.06 & 0.12 & 0.06 & 0.12 \\
\hline Vancomycin & & & 100 & $>8$ & $>8$ & $>8$ & $>8$ \\
\hline
\end{tabular}

${ }^{*}$ Based upon oxacillin susceptibility; ${ }^{\dagger} 5$ vanA and 3 vanB. I intermediate; Max Maximum; $M I C_{50 / 90}$ Minimum inhibitory concentrations (in $\mu \mathrm{g} / \mathrm{mL}$ ) required to inhibit 50\%/90\% of organisms; Min Minimum; No BP No Clinical and Laboratory Standards Institute (or Food and Drug Administration for tigecycline) -approved breakpoints defined; $R$ resistant; $S$ susceptible

activity against VRE with $\mathrm{MIC}_{90} \mathrm{~s}$ of greater than $16 \mu \mathrm{g} / \mathrm{mL}$ and $4 \mu \mathrm{g} / \mathrm{mL}$, respectively.

Antimicrobial activity against the 20 most common organisms isolated from Canadian hospitals (Gram-negative bacilli)

The in vitro activity of various antimicrobials against $E$ coli (including ESBL-producing $E$ coli), $P$ aeruginosa, K pneumoniae, H influenzae, E cloacae, $P$ mirabilis, Serratia marcescens, S maltophilia, Klebsiella oxytoca, Moraxella catarrhalis and A baumannii is displayed in Table 2. For E coli, resistance rates were: TMPSMX 26.6\%, ciprofloxacin and levofloxacin 24.5\% and 23.6\%, respectively, and cefazolin $14.2 \%$ (Table 2). Limited resistance occurred with ceftriaxone $8.9 \%$, gentamicin $10.6 \%$, nitrofurantoin $1.2 \%$, piperacillin-tazobactam $1.3 \%$ and cefepime $2.0 \%$. One hundred per cent susceptibility was observed with ertapenem and meropenem, while $99.8 \%$ of E coli were susceptible to tigecycline (Table 2). Thus, the most active agents against $E$ coli were amikacin, amoxicillin-clavulanate, cefepime, ertapenem, meropenem, piperacillin-tazobactam and tigecycline with $\mathrm{MIC}_{90}$ s of $4 \mu \mathrm{g} / \mathrm{mL}, 8 \mu \mathrm{g} / \mathrm{mL}, 2 \mu \mathrm{g} / \mathrm{mL}, 0.06 \mu \mathrm{g} / \mathrm{mL}$ or less, $0.12 \mu \mathrm{g} / \mathrm{mL}$ or less, $4 \mu \mathrm{g} / \mathrm{mL}$ and $1 \mu \mathrm{g} / \mathrm{mL}$, respectively. ESBL-producing E coli displayed $92.5 \%$ resistance to ciprofloxacin, $67.9 \%$ resistance to TMP-SMX and $58.5 \%$ resistance to gentamicin. All ESBL-producing E coli were susceptible to ertapenem, meropenem, nitrofurantoin and tigecycline, with $\mathrm{MIC}_{90} \mathrm{~s}$ of $0.12 \mu \mathrm{g} / \mathrm{mL}, 0.12 \mu \mathrm{g} / \mathrm{mL}$ or less, $32 \mu \mathrm{g} / \mathrm{mL}$ and $1 \mu \mathrm{g} / \mathrm{mL}$, respectively. The most active agents tested against $P$ aeruginosa were piperacillin-tazobactam, meropenem, colistin (polymyxin E) and amikacin, with $92.7 \%, 87.8 \%, 87.6 \%$ and $85.4 \%$ susceptibility and $\mathrm{MIC}_{90}$ of $64 \mu \mathrm{g} / \mathrm{mL}, 8 \mu \mathrm{g} / \mathrm{mL}, 4 \mu \mathrm{g} / \mathrm{mL}$ and $32 \mu \mathrm{g} / \mathrm{mL}$, respectively (Table 2 ). Resistance with $P$ aeruginosa was high with fluoroquinolones $23.4 \%$ to $25.1 \%$ and gentamicin $20.8 \%$. All agents were active against $H$ influenzae except TMP-SMX, with $12.1 \%$ resistance. For $K$ pneumoniae, resistance rates were: TMP-SMX $8.8 \%$, cefazolin $7.0 \%$, fluoroquinolones $4.2 \%$ to $6.6 \%$, piperacillin-tazobactam $2.0 \%$, tigecycline $1.7 \%$ and ceftriaxone $3.1 \%$. One hundred per cent susceptibility occurred with ertapenem and meropenem as well as $99.6 \%$ with amikacin (Table 2). With E cloacae, resistance rates were: cefazolin $91.0 \%$, ceftriaxone $18.1 \%$, TMP-SMX $8.4 \%$, piperacillintazobactam $9.1 \%$, gentamicin $3.6 \%$, fluoroquinolones $3.0 \%$ to $7.8 \%$ and tigecycline $1.2 \%$. One hundred per cent susceptibility occurred with amikacin, cefepime, ertapenem and meropenem (Table 2). With $P$ mirabilis, resistance rates were: cefazolin 5.0\%, TMP-SMX 9.2\%, fluoroquinolones $7.6 \%$ to $9.2 \%$ and gentamicin $3.4 \%$. One hundred per cent susceptibility occurred with cefepime, ceftriaxone, ertapenem, meropenem and piperacillin-tazobactam (Table 2). With $\mathrm{S}$ marcescens, resistance rates were: cefazolin $99.1 \%$, TMPSMX 2.8\%, fluoroquinolones $4.7 \%$ to $7.5 \%$, ceftriaxone $2.8 \%$, gentamicin $4.7 \%$, and piperacillin-tazobactam $0.9 \%$. With $\mathrm{S}$ marcescens, $100 \%$ susceptibility occurred with cefepime, ertapenem and meropenem, while $99.1 \%$ were susceptible to amikacin (Table 2). The most active agents tested against S maltophilia were TMP-SMX and levofloxacin with $75.5 \%$ and $65.1 \%$ susceptibility, respectively, and $\mathrm{MIC}_{90} \mathrm{~s}$ of $8 \mu \mathrm{g} / \mathrm{mL}$ and $8 \mu \mathrm{g} / \mathrm{mL}$, respectively. The remaining agents demonstrated high rates of resistance $(61.5 \%$ to $97.2 \%)$. Tigecycline was active with $\mathrm{MIC}_{50} \mathrm{~s}$ and $\mathrm{MIC}_{90} \mathrm{~s}$ of $2 \mu \mathrm{g} / \mathrm{mL}$ and $8 \mu \mathrm{g} / \mathrm{mL}$, respectively. All agents were very active against $M$ catarrhalis. With $K$ oxytoca, all agents were very active except cefazolin, with $17.0 \%$ resistance. The most active agents tested against A baumannii were amikacin, gentamicin, levofloxacin and meropenem with $92.0 \%$ susceptibility for all four agents, and $\mathrm{MIC}_{90} \mathrm{~s}$ of $2 \mu \mathrm{g} / \mathrm{mL}$ or less, $1 \mu \mathrm{g} / \mathrm{mL}, 1 \mu \mathrm{g} / \mathrm{mL}$ and $4 \mu \mathrm{g} / \mathrm{mL}$, respectively. Tigecycline was active with $\mathrm{MIC}_{50} \mathrm{~s}$ and $\mathrm{MIC}_{90} \mathrm{~s}$ of $0.5 \mu \mathrm{g} / \mathrm{mL}$ and $2 \mu \mathrm{g} / \mathrm{mL}$, respectively.

\section{DISCUSSION}

The CANWARD study was the first national, prospective surveillance study assessing antimicrobial activity against pathogens from Canadian hospitals, including hospital clinics, 
TABLE 2

Antimicrobial activity against the $\mathbf{2 0}$ most common organisms isolated from Canadian hospitals (Gramnegative bacilli)

\begin{tabular}{|c|c|c|c|c|c|c|c|}
\hline & $\% \mathrm{~S}$ & $\%$ I & $\% \mathbf{R}$ & $\mathrm{MIC}_{50}$ & $\mathrm{MIC}_{90}$ & $\begin{array}{c}\text { Range } \\
\text { Min }\end{array}$ & $\begin{array}{l}\text { Range } \\
\text { Max }\end{array}$ \\
\hline \multicolumn{8}{|c|}{ Escherichia coli $(n=1701)$} \\
\hline Amikacin & 99.5 & 0.4 & 0.1 & $\leq 2$ & 4 & $\leq 2$ & $>64$ \\
\hline $\begin{array}{l}\text { Amoxicllin/ } \\
\text { Clavulanate }\end{array}$ & 90.3 & 8.5 & 1.2 & 4 & 8 & 0.5 & 32 \\
\hline Cefazolin & 82.1 & 3.7 & 14.2 & 2 & 64 & $\leq 0.5$ & $>128$ \\
\hline Cefepime & 95.2 & 2.8 & 2 & $\leq 1$ & 2 & $\leq 0.25$ & $>128$ \\
\hline Cefoxitin & 92.4 & 3.8 & 3.8 & 4 & 8 & $\leq 0.06$ & $>128$ \\
\hline Ceftriaxone & 89.2 & 1.9 & 8.9 & $\leq 1$ & 16 & $\leq 0.25$ & $>256$ \\
\hline Ciprofloxacin & 75.2 & 0.3 & 24.5 & $\leq 0.06$ & $>16$ & $\leq 0.06$ & $>16$ \\
\hline Colistin & No BP & & & 0.5 & 1 & $\leq 0.06$ & $>16$ \\
\hline Ertapenem & 100 & & & $\leq 0.06$ & $\leq 0.06$ & $\leq 0.06$ & 1 \\
\hline Gentamicin & 88.9 & 0.5 & 10.6 & $\leq 0.5$ & 16 & $\leq 0.5$ & $>32$ \\
\hline Levofloxacin & 75.7 & 0.8 & 23.6 & $\leq 0.06$ & 16 & $\leq 0.06$ & $>32$ \\
\hline Meropenem & 100 & & & $\leq 0.12$ & $\leq 0.12$ & $\leq 0.06$ & 0.5 \\
\hline Moxifloxacin & No BP & & & $\leq 0.06$ & $>16$ & $\leq 0.06$ & $>16$ \\
\hline Nitrofurantoin & 95.7 & 3.1 & 1.2 & 16 & 32 & $\leq 0.5$ & $>256$ \\
\hline $\begin{array}{l}\text { Piperacillin/ } \\
\text { Tazobactam }\end{array}$ & 97.6 & 1.1 & 1.3 & 2 & 4 & $\leq 1$ & $>512$ \\
\hline Tigecycline & 99.8 & 0.2 & & 0.25 & 1 & 0.06 & 4 \\
\hline TMP/SMX & 73.4 & & 26.6 & $\leq 0.12$ & $>8$ & $\leq 0.12$ & $>8$ \\
\hline \multicolumn{8}{|c|}{ Extended-spectrum beta-lactamase $E$ coli $(n=53)$} \\
\hline Amikacin & 94.3 & 3.8 & 1.9 & 4 & 16 & $\leq 2$ & $>64$ \\
\hline $\begin{array}{l}\text { Amoxicllin/ } \\
\text { Clavulanate }\end{array}$ & 60.4 & 37.7 & 1.9 & 8 & 16 & 4 & 16 \\
\hline Cefazolin & & & 100 & 128 & $>128$ & 128 & $>128$ \\
\hline Cefepime & 45.3 & 30.2 & 24.5 & 16 & $>32$ & $\leq 1$ & $>32$ \\
\hline Cefoxitin & 92.4 & 5.7 & 1.9 & 8 & 8 & 4 & $>32$ \\
\hline Ceftriaxone & 3.8 & 15.1 & 81.1 & $>64$ & $>64$ & 2 & $>64$ \\
\hline Ciprofloxacin & 7.5 & & 92.5 & $>16$ & $>16$ & $\leq 0.06$ & $>16$ \\
\hline Colistin & No BP & & & 1 & 1 & 0.25 & 2 \\
\hline Ertapenem & 100 & & & $\leq 0.06$ & 0.12 & $\leq 0.06$ & 0.25 \\
\hline Gentamicin & 41.5 & & 58.5 & 32 & $>32$ & $\leq 0.5$ & $>32$ \\
\hline Levofloxacin & 7.5 & & 92.5 & 16 & 32 & $\leq 0.06$ & $>32$ \\
\hline Meropenem & 100 & & & $\leq 0.12$ & $\leq 0.12$ & $\leq 0.12$ & $\leq 0.12$ \\
\hline Moxifloxacin & No BP & & & $>16$ & $>16$ & $\leq 0.06$ & $>16$ \\
\hline Nitrofurantoin & 96.2 & 3.8 & & 16 & 32 & 8 & 32 \\
\hline $\begin{array}{l}\text { Piperacillin/ } \\
\text { Tazobactam }\end{array}$ & 92.4 & 5.7 & 1.9 & 4 & 16 & $\leq 1$ & $>512$ \\
\hline Tigecycline & 100 & & & 0.5 & 1 & 0.25 & 2 \\
\hline TMP/SMX & 32.1 & & 67.9 & $>8$ & $>8$ & $\leq 0.12$ & $>8$ \\
\hline \multicolumn{8}{|c|}{ Pseudomonas aeruginosa $(n=633)$} \\
\hline Amikacin & 85.4 & 7 & 7.6 & 8 & 32 & $\leq 2$ & $>64$ \\
\hline $\begin{array}{l}\text { Amoxicllin/ } \\
\text { Clavulanate }\end{array}$ & No BP & & & $>32$ & $>32$ & 1 & $>32$ \\
\hline Cefazolin & No BP & & & $>128$ & $>128$ & 16 & $>128$ \\
\hline Cefepime & 67.4 & 20.9 & 11.7 & 8 & 32 & $\leq 0.25$ & $>128$ \\
\hline Cefoxitin & No BP & & & $>32$ & $>32$ & 2 & $>32$ \\
\hline Ceftriaxone & 23.9 & 40.9 & 35.2 & 32 & 256 & $\leq 0.25$ & $>256$ \\
\hline Ciprofloxacin & 66 & 10.6 & 23.4 & 0.5 & 16 & $\leq 0.06$ & $>16$ \\
\hline Colistin & 87.6 & & 12.4 & 2 & 4 & 0.5 & $>16$ \\
\hline Ertapenem & No BP & & & 8 & 32 & 0.12 & $>32$ \\
\hline Gentamicin & 60.2 & 19 & 20.8 & 4 & $>32$ & $\leq 0.5$ & $>32$ \\
\hline Levofloxacin & 61.5 & 13.4 & 25.1 & 2 & 16 & $\leq 0.06$ & $>32$ \\
\hline Meropenem & 87.8 & 4.1 & 8.1 & 0.5 & 8 & $\leq 0.06$ & $>64$ \\
\hline Moxifloxacin & No $B P$ & & & 4 & $>16$ & $\leq 0.06$ & $>16$ \\
\hline
\end{tabular}

TABLE 2 - CONTINUED

Antimicrobial activity against the $\mathbf{2 0}$ most common organisms isolated from Canadian hospitals (Gramnegative bacilli)

\begin{tabular}{|c|c|c|c|c|c|c|c|}
\hline & $\% \mathrm{~S}$ & $\%$ I & $\% \mathbf{R}$ & $\mathrm{MIC}_{50}$ & $\mathrm{MIC}_{90}$ & $\begin{array}{c}\text { Range } \\
\text { Min }\end{array}$ & $\begin{array}{c}\text { Range } \\
\text { Max }\end{array}$ \\
\hline \multicolumn{8}{|c|}{ Pseudomonas aeruginosa $(\mathrm{n}=633)$ - CONTINUED } \\
\hline Nitrofurantoin & No BP & & & $>256$ & $>256$ & 16 & $>256$ \\
\hline $\begin{array}{l}\text { Piperacillin/ } \\
\text { Tazobactam }\end{array}$ & 92.7 & & 7.3 & 4 & 64 & $\leq 1$ & $>512$ \\
\hline Tigecycline & No BP & & & $>16$ & $>16$ & 0.25 & $>16$ \\
\hline TMP/SMX & 14.5 & & 85.5 & $>8$ & $>8$ & $\leq 0.12$ & $>8$ \\
\hline \multicolumn{8}{|c|}{ Klebsiella pneumoniae $(n=455)$} \\
\hline Amikacin & 99.6 & & 0.4 & $\leq 2$ & $\leq 2$ & $\leq 2$ & $>64$ \\
\hline $\begin{array}{l}\text { Amoxicllin/ } \\
\text { Clavulanate }\end{array}$ & 93.5 & 5 & 1.5 & 2 & 8 & 1 & $>32$ \\
\hline Cefazolin & 91.2 & 1.8 & 7 & 2 & 8 & $\leq 0.5$ & $>128$ \\
\hline Cefepime & 97.8 & 0.2 & 2 & $\leq 1$ & $\leq 1$ & $\leq 0.25$ & 128 \\
\hline Cefoxitin & 91 & 4.5 & 4.5 & 4 & 8 & 1 & $>32$ \\
\hline Ceftriaxone & 96.2 & 0.7 & 3.1 & $\leq 1$ & $\leq 1$ & $\leq 0.25$ & $>256$ \\
\hline Ciprofloxacin & 92.5 & 0.9 & 6.6 & $\leq 0.06$ & 0.5 & $\leq 0.06$ & $>16$ \\
\hline Colistin & No BP & & & 0.5 & 1 & 0.12 & $>16$ \\
\hline Ertapenem & 100 & & & $\leq 0.06$ & $\leq 0.06$ & $\leq 0.06$ & 2 \\
\hline Gentamicin & 96.7 & 0.4 & 2.9 & $\leq 0.5$ & $\leq 0.05$ & $\leq 0.5$ & $>32$ \\
\hline Levofloxacin & 93.8 & 2 & 4.2 & $\leq 0.06$ & 1 & $\leq 0.06$ & $>32$ \\
\hline Meropenem & 100 & & & $\leq 0.12$ & $\leq 0.12$ & $\leq 0.06$ & 0.25 \\
\hline Moxifloxacin & No $B P$ & & & 0.12 & 1 & $\leq 0.06$ & $>16$ \\
\hline Nitrofurantoin & 35.1 & 33.2 & 31.7 & 64 & 128 & 8 & $>256$ \\
\hline $\begin{array}{l}\text { Piperacillin/ } \\
\text { Tazobactam }\end{array}$ & 96.7 & 1.3 & 2 & 2 & 8 & $\leq 1$ & $>512$ \\
\hline Tigecycline & 94.3 & 4 & 1.7 & 1 & 2 & 0.25 & $>16$ \\
\hline TMP/SMX & 91.2 & & 8.8 & $\leq 0.12$ & 2 & $\leq 0.12$ & $>8$ \\
\hline \multicolumn{8}{|c|}{ Haemophilus influenzae $(n=342)$} \\
\hline $\begin{array}{l}\text { Amoxicillin/ } \\
\text { Clavulanate }\end{array}$ & 99.7 & & 0.3 & 0.5 & 1 & $\leq 0.06$ & 8 \\
\hline Cefepime & 100 & & & $\leq 0.25$ & $\leq 0.25$ & $\leq 0.25$ & 2 \\
\hline Ceftriaxone & 99.7 & & 0.3 & $\leq 0.06$ & $\leq 0.06$ & $\leq 0.06$ & $>4$ \\
\hline Ciprofloxacin & 100 & & & $\leq 0.015$ & $\leq 0.015$ & $\leq 0.015$ & 0.5 \\
\hline Ertapenem & 99.7 & & 0.3 & $\leq 0.03$ & 0.12 & $\leq 0.03$ & $>4$ \\
\hline Gentamicin & No $B P$ & & & 1 & 2 & $\leq 0.5$ & 16 \\
\hline Levofloxacin & 100 & & & $\leq 0.015$ & 0.03 & $\leq 0.015$ & 0.5 \\
\hline Meropenem & 99.7 & & 0.3 & $\leq 0.06$ & 0.12 & $\leq 0.06$ & 2 \\
\hline Moxifloxacin & 100 & & & $\leq 0.015$ & 0.06 & $\leq 0.015$ & 0.5 \\
\hline $\begin{array}{l}\text { Piperacillin/ } \\
\text { Tazobactam }\end{array}$ & 99.7 & & 0.3 & $\leq 1$ & $\leq 1$ & $\leq 1$ & 2 \\
\hline Tigecycline & No BP & & & 0.12 & 0.5 & $\leq 0.03$ & 4 \\
\hline TMP/SMX & 83.5 & 4.4 & 12.1 & $\leq 0.12$ & 4 & $\leq 0.12$ & $>8$ \\
\hline \multicolumn{8}{|c|}{ Enterobacter cloacae $(\mathrm{n}=166)$} \\
\hline Amikacin & 100 & & & $\leq 2$ & $\leq 2$ & $\leq 2$ & 16 \\
\hline $\begin{array}{l}\text { Amoxicillin/ } \\
\text { Clavulanate }\end{array}$ & 8.4 & 20.8 & 70.8 & 32 & $>32$ & 2 & $>32$ \\
\hline Cefazolin & 5.4 & 3.6 & 91 & 128 & $>128$ & 1 & $>128$ \\
\hline Cefepime & 100 & & & $\leq 1$ & 2 & $\leq 0.25$ & 8 \\
\hline Cefoxitin & 48.6 & 8.3 & 43.1 & 16 & $>32$ & 4 & $>32$ \\
\hline Ceftriaxone & 78.3 & 3.6 & 18.1 & $\leq 1$ & $>64$ & $\leq 0.25$ & $>256$ \\
\hline Ciprofloxacin & 91.6 & 0.6 & 7.8 & $\leq 0.06$ & 0.5 & $\leq 0.06$ & $>16$ \\
\hline Colistin & No BP & & & 0.5 & 16 & 0.12 & $>16$ \\
\hline Ertapenem & 100 & & & $\leq 0.06$ & 0.5 & $\leq 0.06$ & 2 \\
\hline Gentamicin & 96.4 & & 3.6 & $\leq 0.5$ & 1 & $\leq 0.5$ & $>32$ \\
\hline Levofloxacin & 92.8 & 4.2 & 3 & $\leq 0.06$ & 1 & $\leq 0.06$ & 32 \\
\hline Meropenem & 100 & & & $\leq 0.12$ & $\leq 0.12$ & $\leq 0.06$ & 0.5 \\
\hline
\end{tabular}


TABLE 2 - CONTINUED

Antimicrobial activity against the $\mathbf{2 0}$ most common organisms isolated from Canadian hospitals (Gramnegative bacilli)

\begin{tabular}{|c|c|c|c|c|c|c|c|}
\hline & $\% \mathrm{~S}$ & $\%$ I & $\% \mathbf{R}$ & $\mathrm{MIC}_{50}$ & $\mathrm{MIC}_{90}$ & $\begin{array}{l}\text { Range } \\
\text { Min }\end{array}$ & $\begin{array}{l}\text { Range } \\
\text { Max }\end{array}$ \\
\hline \multicolumn{8}{|c|}{ Enterobacter cloacae $(\mathrm{n}=166)$ - CONTINUED } \\
\hline Moxifloxacin & No $B P$ & & & 0.12 & 0.5 & $\leq 0.06$ & $>16$ \\
\hline Nitrofurantoin & 54.2 & 38.9 & 6.9 & 32 & 64 & 16 & 256 \\
\hline $\begin{array}{l}\text { Piperacillin/ } \\
\text { Tazobactam }\end{array}$ & 82.5 & 8.4 & 9.1 & 2 & 64 & $\leq 1$ & 512 \\
\hline Tigecycline & 93.4 & 5.4 & 1.2 & 1 & 1 & 0.25 & 16 \\
\hline TMP/SMX & 91.6 & & 8.4 & $\leq 0.12$ & 1 & $\leq 0.12$ & $>8$ \\
\hline \multicolumn{8}{|c|}{ Proteus mirabilis $(\mathrm{n}=119)$} \\
\hline Amikacin & 99.2 & 0.8 & & 4 & 8 & $\leq 2$ & 32 \\
\hline $\begin{array}{l}\text { Amoxicillin/ } \\
\text { Clavulanate }\end{array}$ & 97.1 & & 2.9 & 1 & 4 & 0.5 & 32 \\
\hline Cefazolin & 86.6 & 8.4 & 5 & 8 & 16 & 1 & 64 \\
\hline Cefepime & 100 & & & $\leq 1$ & $\leq 1$ & $\leq 0.25$ & 2 \\
\hline Cefoxitin & 91.2 & 8.8 & & 4 & 8 & 2 & 16 \\
\hline Ceftriaxone & 100 & & & $\leq 1$ & $\leq 1$ & $\leq 0.25$ & 4 \\
\hline Ciprofloxacin & 82.4 & 8.4 & 9.2 & $\leq 0.06$ & 2 & $\leq 0.06$ & $>16$ \\
\hline Colistin & No BP & & & $>16$ & $>16$ & $>16$ & $>16$ \\
\hline Ertapenem & 100 & & & $\leq 0.06$ & $\leq 0.06$ & $\leq 0.06$ & 0.12 \\
\hline Gentamicin & 95.8 & 0.8 & 3.4 & 1 & 2 & $\leq 0.5$ & $>32$ \\
\hline Levofloxacin & 88.2 & 4.2 & 7.6 & 0.12 & 4 & $\leq 0.06$ & $>32$ \\
\hline Meropenem & 100 & & & $\leq 0.12$ & $\leq 0.12$ & $\leq 0.06$ & 0.25 \\
\hline Moxifloxacin & No BP & & & 0.5 & 16 & 0.12 & $>16$ \\
\hline Nitrofurantoin & & 5.9 & 94.1 & 128 & 128 & 64 & 256 \\
\hline $\begin{array}{l}\text { Piperacillin/ } \\
\text { Tazobactam }\end{array}$ & 100 & & & $\leq 1$ & $\leq 1$ & $\leq 1$ & 2 \\
\hline Tigecycline & 10.1 & 35.3 & 54.6 & 8 & 8 & 1 & 16 \\
\hline TMP/SMX & 90.8 & & 9.2 & $\leq 0.12$ & 2 & $\leq 0.12$ & $>8$ \\
\hline \multicolumn{8}{|c|}{ Serratia marcescens $(n=108)$} \\
\hline Amikacin & 99.1 & 0.9 & & $\leq 2$ & 4 & $\leq 2$ & 32 \\
\hline $\begin{array}{l}\text { Amoxicillin/ } \\
\text { Clavulanate }\end{array}$ & 2.6 & 30.8 & 66.6 & 32 & $>32$ & 4 & $>32$ \\
\hline Cefazolin & 0.9 & & 99.1 & $>128$ & $>128$ & 2 & $>128$ \\
\hline Cefepime & 100 & & & $\leq 1$ & $\leq 1$ & $\leq 0.25$ & 8 \\
\hline Cefoxitin & 7.7 & 53.8 & 38.5 & 16 & $>32$ & 4 & $>32$ \\
\hline Ceftriaxone & 92.5 & 4.7 & 2.8 & $\leq 1$ & $\leq 1$ & $\leq 0.25$ & $>64$ \\
\hline Ciprofloxacin & 88.8 & 3.7 & 7.5 & 0.12 & 2 & $\leq 0.06$ & 16 \\
\hline Colistin & No BP & & & $>16$ & $>16$ & $>16$ & $>16$ \\
\hline Ertapenem & 100 & & & $\leq 0.06$ & $\leq 0.06$ & $\leq 0.06$ & 0.5 \\
\hline Gentamicin & 91.6 & 3.7 & 4.7 & $\leq 0.5$ & 1 & $\leq 0.5$ & $>32$ \\
\hline Levofloxacin & 90.6 & 4.7 & 4.7 & 0.12 & 2 & $\leq 0.06$ & 16 \\
\hline Meropenem & 100 & & & $\leq 0.12$ & $\leq 0.12$ & $\leq 0.06$ & 2 \\
\hline Moxifloxacin & No BP & & & 0.25 & 4 & $\leq 0.06$ & $>16$ \\
\hline Nitrofurantoin & & 2.6 & 97.4 & 256 & $>256$ & 64 & $>256$ \\
\hline $\begin{array}{l}\text { Piperacillin/ } \\
\text { Tazobactam }\end{array}$ & 94.4 & 4.7 & 0.9 & 2 & 8 & $\leq 1$ & 128 \\
\hline Tigecycline & 61.7 & 32.7 & 5.6 & 2 & 4 & 0.12 & $>16$ \\
\hline TMP/SMX & 97.2 & & 2.8 & 0.5 & 1 & $\leq 0.12$ & 8 \\
\hline \multicolumn{8}{|c|}{ Stenotrophomonas maltophilia $(n=107)$} \\
\hline Amikacin* & 16 & 8.5 & 75.5 & $>64$ & $>64$ & $\leq 2$ & $>64$ \\
\hline $\begin{array}{l}\text { Amoxicillin/ } \\
\text { Clavulanate }\end{array}$ & No BP & & & $>32$ & $>32$ & 4 & $>32$ \\
\hline Cefazolin & No BP & & & $>128$ & $>128$ & 128 & $>128$ \\
\hline Cefepime* & 4.7 & 6.6 & 88.7 & 64 & 128 & $\leq 0.25$ & $>128$ \\
\hline Cefoxitin & No BP & & & $>32$ & $>32$ & 8 & $>32$ \\
\hline Ceftriaxone* & 0.9 & 1.9 & 97.2 & 256 & $>256$ & 8 & $>256$ \\
\hline
\end{tabular}

Continued in next column
TABLE 2 - CONTINUED

Antimicrobial activity against the 20 most common organisms isolated from Canadian hospitals (Gramnegative bacilli)

\begin{tabular}{|c|c|c|c|c|c|c|c|}
\hline & $\% \mathrm{~S}$ & $\%$ I & $\% \mathrm{R}$ & $\mathrm{MIC}_{50}$ & $\mathrm{MIC}_{90}$ & $\begin{array}{l}\text { Range } \\
\text { Min }\end{array}$ & $\begin{array}{l}\text { Range } \\
\text { Max }\end{array}$ \\
\hline \multicolumn{8}{|c|}{ Stenotrophomonas maltophilia $(n=107)$ - CONTINUED } \\
\hline Ciprofloxacin* & 6.6 & 24.5 & 68.9 & 4 & $>16$ & $\leq 0.06$ & $>16$ \\
\hline Colistin* & 26.9 & 11.6 & 61.5 & 8 & $>16$ & 0.25 & $>16$ \\
\hline Ertapenem & No BP & & & $>32$ & $>32$ & 0.12 & $>32$ \\
\hline Gentamicin* & 17 & 4.7 & 78.3 & 32 & $>32$ & $\leq 0.5$ & $>32$ \\
\hline Levofloxacin & 65.1 & 14.2 & 20.7 & 2 & 8 & $\leq 0.06$ & $>32$ \\
\hline Meropenem* & 5.7 & 1.9 & 92.4 & $>64$ & $>64$ & $\leq 0.06$ & $>64$ \\
\hline Moxifloxacin & No BP & & & 1 & 8 & 0.12 & $>16$ \\
\hline Nitrofurantoin & No BP & & & $>256$ & $>256$ & 32 & $>256$ \\
\hline $\begin{array}{l}\text { Piperacillin/ } \\
\text { Tazobactam* }\end{array}$ & 3.8 & 14.1 & 82.1 & 256 & $>512$ & 16 & $>512$ \\
\hline Tigecycline & No BP & & & 2 & 8 & 0.25 & 16 \\
\hline TMP/SMX & 75.5 & & 24.5 & 1 & 8 & $\leq 0.12$ & $>8$ \\
\hline \multicolumn{8}{|c|}{ Klebsiella oxytoca $(n=100)$} \\
\hline Amikacin & 100 & & & $\leq 2$ & $\leq 2$ & $\leq 2$ & 16 \\
\hline $\begin{array}{l}\text { Amoxicillin/ } \\
\text { Clavulanate }\end{array}$ & 96.9 & 3.1 & & 2 & 4 & 1 & 16 \\
\hline Cefazolin & 60 & 23 & 17 & 8 & 32 & $\leq 0.5$ & $>128$ \\
\hline Cefepime & 99 & 1 & & $\leq 1$ & $\leq 1$ & $\leq 0.25$ & 16 \\
\hline Cefoxitin & 96.9 & 3.1 & & 2 & 4 & 1 & 16 \\
\hline Ceftriaxone & 94 & 6 & & $\leq 1$ & $\leq 1$ & $\leq 0.25$ & 32 \\
\hline Ciprofloxacin & 95 & 2 & 3 & $\leq 0.06$ & 0.12 & $\leq 0.06$ & 16 \\
\hline Colistin & No BP & & & 0.5 & 1 & 0.25 & 2 \\
\hline Ertapenem & 100 & & & $\leq 0.06$ & $\leq 0.06$ & $\leq 0.06$ & $\leq 0.06$ \\
\hline Gentamicin & 97 & 2 & 1 & $\leq 0.5$ & $\leq 0.5$ & $\leq 0.5$ & $>32$ \\
\hline Levofloxacin & 96 & 2 & 2 & $\leq 0.06$ & 0.12 & $\leq 0.06$ & 8 \\
\hline Meropenem & 100 & & & $\leq 0.12$ & $\leq 0.12$ & $\leq 0.06$ & 0.12 \\
\hline Moxifloxacin & No BP & & & 0.12 & 0.25 & $\leq 0.06$ & $>16$ \\
\hline Nitrofurantoin & 75 & 21.9 & 3.1 & 32 & 64 & 8 & 128 \\
\hline $\begin{array}{l}\text { Piperacillin/ } \\
\text { Tazobactam }\end{array}$ & 90 & 1 & 9 & 2 & 16 & $\leq 1$ & $>512$ \\
\hline Tigecycline & 99 & & 1 & 0.5 & 2 & 0.25 & 8 \\
\hline TMP/SMX & 95 & & 5 & $\leq 0.12$ & $\leq 0.12$ & $\leq 0.12$ & $>8$ \\
\hline \multicolumn{8}{|c|}{ Moraxella catarrhalis $(n=93)$} \\
\hline \multicolumn{8}{|c|}{ Amikacin } \\
\hline $\begin{array}{l}\text { Amoxicillin/ } \\
\text { Clavulanate }\end{array}$ & No BP & & & 0.12 & 0.25 & $\leq 0.06$ & 0.5 \\
\hline \multicolumn{8}{|l|}{ Cefazolin } \\
\hline \multicolumn{8}{|l|}{ Cefepime } \\
\hline \multicolumn{8}{|l|}{ Cefoxitin } \\
\hline Ceftriaxone & No BP & & & 0.25 & 1 & $\leq 0.06$ & 1 \\
\hline Ciprofloxacin & No BP & & & $\leq 0.06$ & $\leq 0.06$ & $\leq 0.06$ & 0.12 \\
\hline \multicolumn{8}{|l|}{ Colistin } \\
\hline Ertapenem & No BP & & & $\leq 0.06$ & $\leq 0.06$ & $\leq 0.06$ & 0.12 \\
\hline \multicolumn{8}{|l|}{ Gentamicin } \\
\hline Levofloxacin & No BP & & & $\leq 0.06$ & $\leq 0.06$ & $\leq 0.06$ & 0.12 \\
\hline Meropenem & No BP & & & $\leq 0.06$ & $\leq 0.06$ & $\leq 0.06$ & $\leq 0.06$ \\
\hline Moxifloxacin & No BP & & & $\leq 0.06$ & $\leq 0.06$ & $\leq 0.06$ & 0.25 \\
\hline \multicolumn{8}{|l|}{ Nitrofurantoin } \\
\hline $\begin{array}{l}\text { Piperacillin/ } \\
\text { Tazobactam }\end{array}$ & No BP & & & $\leq 1$ & $\leq 1$ & $\leq 1$ & $\leq 1$ \\
\hline Tigecycline & No BP & & & 0.12 & 0.25 & 0.06 & 0.5 \\
\hline TMP/SMX & No BP & & & $\leq 0.12$ & 2 & $\leq 0.12$ & $>8$ \\
\hline
\end{tabular}


TABLE 2 - CONTINUED

Antimicrobial activity against the 20 most common organisms isolated from Canadian hospitals (Gramnegative bacilli)

\begin{tabular}{|c|c|c|c|c|c|c|c|}
\hline & $\% \mathrm{~S}$ & $\%$ I & $\% \mathbf{R}$ & $\mathrm{MIC}_{50}$ & $\mathrm{MIC}_{90}$ & $\begin{array}{l}\text { Range } \\
\text { Min }\end{array}$ & $\begin{array}{c}\text { Range } \\
\text { Max }\end{array}$ \\
\hline \multicolumn{8}{|c|}{ Acinetobacter baumannii $(n=25)$} \\
\hline Amikacin & 92 & & 8 & $\leq 2$ & $\leq 2$ & $\leq 2$ & $>64$ \\
\hline $\begin{array}{l}\text { Amoxicillin/ } \\
\text { Clavulanate }\end{array}$ & No BP & & & 8 & 32 & 2 & $>32$ \\
\hline Cefazolin & No BP & & & $>128$ & $>128$ & 64 & $>128$ \\
\hline Cefepime & 84 & 8 & 8 & 4 & 16 & $\leq 1$ & $>128$ \\
\hline Cefoxitin & No BP & & & $>32$ & $>32$ & 8 & $>32$ \\
\hline Ceftriaxone & 24 & 68 & 8 & 16 & 32 & 4 & $>256$ \\
\hline Ciprofloxacin & 88 & & 12 & 0.25 & 4 & 0.12 & 32 \\
\hline Colistin & No BP & & & 1 & 2 & 1 & 2 \\
\hline Ertapenem & No BP & & & 4 & 8 & 2 & $>32$ \\
\hline Gentamicin & 92 & & 8 & $\leq 0.5$ & 1 & $\leq 0.5$ & $>32$ \\
\hline Levofloxacin & 92 & & 8 & 0.25 & 1 & $\leq 0.06$ & $>16$ \\
\hline Meropenem & 92 & & 8 & 0.5 & 4 & $\leq 0.12$ & 32 \\
\hline Moxifloxacin & No BP & & & 0.12 & 0.5 & $\leq 0.06$ & 8 \\
\hline Nitrofurantoin & No BP & & & $>256$ & $>256$ & 256 & $>256$ \\
\hline $\begin{array}{l}\text { Piperacillin/ } \\
\text { Tazobactam }\end{array}$ & 76 & 12 & 12 & 4 & $>128$ & $\leq 1$ & $>512$ \\
\hline Tigecycline & No BP & & & 0.5 & 2 & 0.12 & 4 \\
\hline TMP/SMX & 84 & & 16 & $\leq 0.12$ & $>8$ & $\leq 0.12$ & $>8$ \\
\hline \multicolumn{8}{|c|}{$\begin{array}{l}{ }^{*} \text { Non-Enterobacteriaceae breakpoints used. Colistin (polymyxin E); } \\
\text { I Intermediate; Max Maximum; MIC }{ }_{50 / 90} \text { Minimum inhibitory concentrations (in } \\
\mu \mathrm{g} / \mathrm{mL} \text { ) required to inhibit } 50 \% / 90 \% \text { of organisms; Min Minimum; No BP No } \\
\text { Clinical and Laboratory Standards Institute (or Food and Drug Administration for } \\
\text { tigecycline) -approved breakpoints defined; R Resistant; S Susceptible; TMP- } \\
\text { SMX Trimethoprim-sulfamethoxazole }\end{array}$} \\
\hline
\end{tabular}

emergency rooms, medical and surgical wards, and intensive care units. A total of 7881 organisms were obtained between January 1, 2007, and December 31, 2007, inclusive. Of the 7881 organisms, $6885(87.4 \%)$ represented the 20 most common organisms isolated from hospitals in Canada and underwent antimicrobial susceptibility testing.

The most active agents (based upon MIC data only) against the 3178 Gram-positive organisms tested were vancomycin, linezolid, daptomycin, tigecycline, dalbavancin and telavancin (Table 1). It should be mentioned that listing agents as most active based solely upon MIC is not accurate because potency depends both upon the agent's pharmacokinetics as well as in vitro susceptibility (ie, pharmacodynamics). Vancomycin was active against MSSA and MRSA with $\mathrm{MIC}_{90^{5}}$ of $1 \mu \mathrm{g} / \mathrm{mL}$ and $1 \mu \mathrm{g} / \mathrm{mL}$, respectively. Only six of 1095 (0.55\%) MSSA and four of 385 (1.0\%) MRSA demonstrated vancomycin MICs of $2 \mu \mathrm{g} / \mathrm{mL}$. No MSSA or MRSA with vancomycin MICs of $4 \mu \mathrm{g} / \mathrm{mL}$ or greater were obtained. This is consistent with previous data that has reported that vancomycin continues to be active against MSSA and MRSA in Canada $(4,9,11)$. It must however be stated that no population analysis profiling was performed on any MRSA to assess for heteroresistant vancomycin-intermediate $S$ aureus. Against MSSE and MRSE, vancomycin was less active compared with MSSA and MRSA. The $\mathrm{MIC}_{90} \mathrm{~s}$ for both MSSE and MRSE were $2 \mu \mathrm{g} / \mathrm{mL}$. This reduced vancomycin activity against MSSE and MRSE versus
MSSA and MRSA has also been previously documented $(9,16)$. In this study, as well as with previous data, vancomycin continues to be very active against all Streptococcus species, with all isolates displaying MICs of $1 \mu \mathrm{g} / \mathrm{mL}$ or less $(9,17)$. Vancomycin was less active against $E$ faecalis and $E$ faecium with $0 \%$ and $11.7 \%$ of strains resistant, respectively. As has been reported elsewhere, the predominant VRE genotype in North America continues to be vanA $(4,7)$.

Linezolid was active against MSSA and MRSA with 100\% of isolates demonstrating susceptibility with MICs $4 \mu \mathrm{g} / \mathrm{mL}$ or less (Table 1). No difference in linezolid activity was observed between HA-MRSA and CA-MRSA. Linezolid was more active against MSSE and MRSE in comparison with MSSA and MRSA, with all isolates demonstrating linezolid MICs of $1 \mu \mathrm{g} / \mathrm{mL}$ or less (Table 1). Linezolid's continued excellent activity against MSSA/MRSA and MSSE/MRSE has been previously documented $(11,16,17)$. As has been previously documented, linezolid continues to be active against Streptococcus species with all isolates displaying MICs of $2 \mu \mathrm{g} / \mathrm{mL}$ or less $(11,17)$. Linezolid was less active against $E$ faecalis and $E$ faecium, with $1.3 \%$ and $8.6 \%$ of strains demonstrating intermediate resistance, respectively. This rate of linezolid resistance in E faecium is consistent with previous reports (17-19).

Daptomycin was active against MSSA and MRSA with 100\% of isolates demonstrating susceptibility, with MICs of $1 \mu \mathrm{g} / \mathrm{mL}$ or less (Table 1). No difference in daptomycin activity was observed between HA-MRSA and CA-MRSA. Daptomycin was equally active against MSSE and MRSE compared with MSSA and MRSA, with all isolates demonstrating daptomycin MICs of $0.25 \mu \mathrm{g} / \mathrm{mL}$ or less. Daptomycin's excellent activity against MSSA/ MRSA and MSSE/MRSE has been previously documented $(11,16)$. As has been previously reported $(11,16)$, daptomycin was active against Streptococcus species with isolates displaying MICs of $0.12 \mu \mathrm{g} / \mathrm{mL}$ or less. Daptomycin was active against $E$ faecalis, E faecium and VRE, with 100\% susceptibility and all isolates displaying MICs of $2 \mu \mathrm{g} / \mathrm{mL}$ or less (Table 1). Daptomycin-resistant enterococci species continue to be rare (18) and have not been documented in Canada. From these data, it is clear daptomycin is a very active agent against all Gram-positive organisms causing infections in Canadian hospitals.

Tigecycline was active against MSSA and MRSA with 100\% of isolates demonstrating susceptibility, with MICs of $0.5 \mu \mathrm{g} / \mathrm{mL}$ or less (Table 1). No difference in tigecycline activity was observed between HA-MRSA and CA-MRSA. Tigecycline was equally active against MSSE and MRSE compared with MSSA and MRSA, with all isolates demonstrating tigecycline MICs of $0.5 \mu \mathrm{g} / \mathrm{mL}$ or less. Tigecycline's excellent activity against MSSA/ MRSA and MSSE/MRSE has been previously documented $(11,19)$. As has been previously reported, tigecycline was very active against Streptococcus species, with all isolates displaying MICs of $0.12 \mu \mathrm{g} / \mathrm{mL}$ or less $(11,19)$. Tigecycline was very active against $E$ faecalis, E faecium and VRE, with all isolates displaying MICs of $0.5 \mu \mathrm{g} / \mathrm{mL}$ or less (Table 1). From these data, it is clear tigecycline is a very active agent against all Gram-positive organisms causing infections in Canadian hospitals.

Dalbavancin was active against MSSA and MRSA with $100 \%$ of isolates demonstrating MICs of $0.25 \mu \mathrm{g} / \mathrm{mL}$ or less (Table 1). No difference in dalbavancin activity was observed between HA-MRSA and CA-MRSA. Dalbavancin was equally active against MSSE and MRSE, with all isolates demonstrating 
MICs of $0.12 \mu \mathrm{g} / \mathrm{mL}$ or less. Dalbavancin's excellent activity against MSSA/MRSA and MSSE/MRSE has been previously documented $(11,20)$. As has been previously reported $(11,20)$, dalbavancin was active against Streptococcus species with isolates displaying MICs of $0.12 \mu \mathrm{g} / \mathrm{mL}$ or less. Dalbavancin was active against $E$ faecalis, but displayed less activity against E faecium and VRE (Table 1).

Telavancin was active against MSSA and MRSA with $100 \%$ of isolates demonstrating MICs of $1 \mu \mathrm{g} / \mathrm{mL}$ or less (Table 1 ). No difference in telavancin activity was observed between HA-MRSA and CA-MRSA. Telavancin was equally active against MSSE and MRSE, with all isolates demonstrating MICs of $0.25 \mu \mathrm{g} / \mathrm{mL}$ or less. Telavancin's excellent activity against MSSA/MRSA and MSSE/MRSE has been previously documented $(20,21)$. As has been previously reported (21), telavancin was active against Streptococcus species with isolates displaying MICs of $0.12 \mu \mathrm{g} / \mathrm{mL}$ or less. Telavancin was active against $E$ faecalis, but displayed less activity against $E$ faecium and VRE (Table 1). It has been previously documented that telavancin is active against $\operatorname{VanB}$ Enterococcus species, but not VanA Enterococcus species (21).

The most active (based on MIC only) agents against the 3507 Gram-negative bacilli obtained from Canadian hospitals were amikacin, cefepime, ertapenem (not $P$ aeruginosa), meropenem, piperacillin-tazobactam and tigecycline (not $P$ aeruginosa) (Table 2). Amikacin was very active against $E$ coli (including ESBL-producing strains) with $99.5 \%$ of strains testing susceptible with an $\mathrm{MIC}_{90}$ of $4 \mu \mathrm{g} / \mathrm{mL}$. Likewise, amikacin proved to be very active against all other Enterobacteriaceae tested (Table 2). Against $P$ aeruginosa, amikacin proved to be one of the most active agents tested, with $85.4 \%$ of strains testing susceptible with $\mathrm{MIC}_{90}$ of $32 \mu \mathrm{g} / \mathrm{mL}$. Against A baumannii, amikacin $P$ aeruginosa was very active with $92.0 \%$ of strains being susceptible with $\mathrm{MIC}_{90}$ of $2 \mu \mathrm{g} / \mathrm{mL}$ or less. The excellent activity of amikacin against both Enterobacteriaceae as well as nonfermenters isolated from patients in hospitals, including in the intensive care unit, is not surprising because the reduced usage of aminoglycosides in favour of fluoroquinolones over the past 15 years has resulted in maintained activity of aminoglycosides in the setting on increasing fluoroquinolone resistance $(4,19,22)$. Thus, amikacin represents a potential option for the treatment of infections caused by Gram-negative bacilli resistant to other less toxic agents.

In the present study, we reported that cefepime, ertapenem, meropenem and piperacillin-tazobactam were very active against Gram-negative bacilli isolated from patients in Canadian hospitals. These agents were active against Enterobacteriaceae including against E coli (only ertapenem and meropenem were active against ESBL-producing strains). Against $P$ aeruginosa, resistance was piperacillin-tazobactam $7.3 \%$, meropenem $8.1 \%$ and cefepime $11.7 \%$. Previous investigators have reported the ongoing excellent activity of these agents versus Gram-negative bacilli isolated from hospitalized patients $(4,19,22)$. Colistin was found to be very active against E coli (including ESBL strains) with $\mathrm{MIC}_{90}$ of $1 \mu \mathrm{g} / \mathrm{mL}$. Colistin was also very active against Klebsiella species, E cloacae and $P$ mirabilis. Against $P$ aeruginosa, resistance to colistin was $12.4 \%$ with an $\mathrm{MIC}_{90}$ of $4 \mu \mathrm{g} / \mathrm{mL}$ (Table 2). Against A baumannii, colistin was also very active, with an $\mathrm{MIC}_{90}$ of $2 \mu \mathrm{g} / \mathrm{mL}$ (Table 2). These data are consistent with other reports of the promising potential of colistin for Gram-negative bacilli such as $P$ aeruginosa and A baumannii $(23,24)$.

Tigecycline demonstrated $99.8 \%$ susceptibility versus $E$ coli (100\% versus ESBL-producing strains) and was also active against other Enterobacteriaceae including $\mathrm{K}$ pneumoniae, E cloacae, S marcescens and Koxytoca (Table 2). Tigecycline was not active against $P$ mirabilis and $P$ aeruginosa. Tigecycline also proved to be active against $S$ maltophilia and A baumannii organisms frequently resistant to other antimicrobial classes (Table 2). The activity of tigecycline against Gram-negative bacilli (with the exception of $P$ aeruginosa) has been previously reported and supports the potential to use this agent for the treatment of infections caused by non-Pseudomonas Gramnegative bacilli in hospitalized patients $(11,19)$.

The present study has several limitations, including the fact that we can not be certain that all clinical specimens represented active infection. In the CANWARD study, we asked centres to obtain 'clinically significant' specimens from patients with a presumed infectious disease. Although all of the isolates may not represent actual infection from patients, we believe that most do because we excluded all surveillance swabs and duplicate swabs, as well as eye, ear, nose and throat swabs and genital cultures. In addition, we do not have admission date data for each patient/clinical specimen, thus were not able to provide a more accurate description of community versus nosocomial onset. Finally, susceptibility testing was not performed for all antimicrobial agents due to lack of space on the susceptibility panels utilized. It is recognized that data on antimicrobials such a ceftazidime, imipenem, tobramycin and others would be beneficial, because different hospital formularies stock these and other antimicrobials not tested in this study.

\section{CONCLUSIONS}

The most active agents versus Gram-positive cocci from Canadian hospitals were vancomycin, linezolid, daptomycin, tigecycline, dalbavancin and telavancin. The most active agents versus Gram-negative bacilli from Canadian hospitals were amikacin, cefepime, ertapenem (not $P$ aeruginosa), meropenem, piperacillin-tazobactam and tigecycline (not $P$ aeruginosa). Colistin was very active against $P$ aeruginosa and A baumannii.

ACKNOWLEDGEMENTS: This paper was presented in part at the 48th Interscience Conference on Antimicrobial Agents and Chemotherapy-ICAAC (2008) in Washington, DC. Funding for the CANWARD 2007 study was provided in part by the University of Manitoba, Health Sciences Center in Winnipeg, National Microbiology Laboratory-Health Canada, Abbott, Affinium Inc, Astellas, Bayer, Janssen Ortho Inc, Merck, Oryx, Pfizer Canada, TaiGen, Targanta and Wyeth Inc. Special thanks to Nancy Laing, Barb Weshnoweski, Ravi Vashisht, Lisa Bittner and Haley Butcher for technological assistance. The authors thank M Tarka for expert secretarial assistance. The authors thank the investigators and laboratory site staff at each medical centre that participated in the CANWARD 2007 study: Vancouver Hospital, Vancouver, British Columbia - Dr D Roscoe; University of Alberta Hospitals, Edmonton, Alberta - Dr R Rennie; Royal University Hospital, Saskatoon, Sascatchewan - Dr J Blondeau; Health Sciences Centre, Winnipeg, Manitoba - Drs D Hoban and G Zhanel; Mount Sinai Hospital, Toronto, Ontario - Dr S Poutanen; 
Children's Hospital of Eastern Ontario, Ottawa, Ontario - Dr F Chan; London Health Sciences Centre, London, Ontario - Dr Z Hussain; St Joseph's Hospital, Hamilton, Ontario - Dr C Lee; Hopital Maisonneuve-Rosemont, Montreal, Quebec - Dr M Laverdiere; Montreal General Hospital, Montreal, Quebec - Dr V Loo; Royal Victoria Hospital, Montreal, Quebec - Dr V Loo; QEII Health Sciences Centre, Halifax, Nova Scotia - Drs K Forward and R Davidson. CANWARD data are also displayed at www.can-r.ca, the official Web site of the Canadian Antimicrobial Resistance Alliance (CARA).

\section{REFERENCES}

1. Anonymous. National nosocomial infections surveillance (NNIS) system report, data summary from January 1992 through June 2003, issued August 2003. Am J Infect Cont 2003;31:481-98.

2. Lockhart SR, Abramson MA, Beekman SE, et al. Antimicrobial resistance among gram-negative bacilli as causes of infections in intensive care unit patients in the United States between 1993 . 2004. J Clin Microbiol 2007;45:3352-59.

3. Rubinstein E, Zhanel GG. Anti-infectives research and development problems challenges and solutions: The clinical practitioner perspective. Lancet Infect Dis 2007;7:69-70.

4. Zhanel GG, DeCorby M, Laing N, et al. Antimicrobial-resistant pathogens in intensive care units in Canada: Results of the Canadian National Intensive Care Unit (CAN-ICU) Study, 2005/2006. Antimicrob Agents Chemother 2008;52:1430-37.

5. Chen DK, McGeer A, de Azavedo JC, Low DE. Decreased susceptibility of Streptococcus pneumoniae to fluoroquinolones in Canada. N Engl J Med 1999;341:233-39.

6. Whitney CG, Farley MM, Hadler J. Increasing prevalence of multi-drug resistant Streptococcus pneumoniae in the United States. N Engl J Med 2000;343:1817-24.

7. Zhanel GG, Laing NM, Nichol KA, et al. Antibiotic activity against urinary tract infection (UTI) isolates of vancomycinresistant enterococci (VRE): Results from the 2002 North America Vancomycin Resistant Enterococci Susceptibility Study (NAVRESS). J Antimicrob Chemother 2004;52:382-88.

8. Chambers HF. Community-associated MRSA-resistance and virulence converge. N Engl J Med 2005;352:1485-88.

9. Mulvey MR, MacDougall L, Cholin B, et al. Community-associated methicillin-resistant Staphylococcus aureus. Canada Emerg Infect Dis 2005;11:844-50.

10. Zhanel GG, DeCorby M, Nichol KA, et al. Characterization of MRSA, VRE and ESBL-producing E. coli in Intensive Care Units in Canada: Results of the Canadian National Intensive Care Unit (CAN-ICU) Study, 2005/2006. Can J Infect Dis Med Microbiol 2008;19(3):243-49.
11. Zhanel GG, DeCorby M, Nichol KA, et al. Antimicrobial susceptibility of 3931 organisms isolated from intensive care units in Canada: Canadian National Intensive Care Unit Study, 2005/2006. Diagn Microbiol Infect Dis 2008;62:67-80.

12. Zhanel GG, Karlowsky JA, DeCorby M, et al. Prevalence of antimicrobial-resistant pathogens in Canadian hospitals: Results of the Canadian Ward Surveillance Study (CANWARD 2007). Can J Infect Dis Med Microbiol 2009;20(Suppl A):9A-19A.

13. Clinical and Laboratory Standards Institute. Performance standards for antimicrobial susceptibility testing: 16th informational supplement document M100-S16. CLSI/NCCLS M100-S15. Clinical and Laboratory Standards Institute, Wayne, PA. 2006.

14. Mulvey MR, Bryce E, Boyd D, et al. Ampler class A extendedspectrum beta-lactamase producing Escherichia coli and Klebsiella spp. in Canadian Hospitals. Antimicrob Agents Chemother 2004:48:1204-14.

15. Mulvey MR, Chiu L, Ismail J, et al. Development of a Canadian standardized protocol for subtyping methicillin-resistant Staphylococcus aureus using pulsed-field gel electrophoresis. J Clin Microbiol 2001;39:3481-85.

16. Pfaller MA, Sader HS, Jones RN. Evaluation of the in vitro activity of daptomycin against 19,615 clinical isolates of gram-positive cocci collected in North American hospitals (2002-2005). Diagn Microbiol Infect Dis 2007;57:459-65.

17. Jones RN, Fritsche TR, Sader HS, et al. LEADER surveillance program results for 2006: An activity and spectrum analysis of linezolid using clinical isolates from the United States (50 medical centers). Diagn Microbiol Infect Dis 2007;59:309-17.

18. Deshpande LM, Fritsche TR, Moet GJ, et al. Antimicrobial resistance and molecular epidemiology of vancomycin-resistant enterococci from North America and Europe: A report from the SENTRY antimicrobial surveillance program. Diagn Microbiol Infect Dis 2007;58:163-70.

19. Waites KB, Duffy LB, Dowzicky MJ. Antimicrobial susceptibility among pathogens collected from hospitalized patients in the United States and in vitro activity of tigecycline a new glycylcycline antimicrobial. Antimicrob Agents Chemother 2006;50:3479-84.

20. Zhanel GG, Trapp S, Gin AS, et al. Dalbavancin and telavancin: Novel lipoglycopeptides for the treatment of gram-positive infections. Expert Rev Anti Infect Ther 2008;6:67-81.

21. Krause KM, Renelli M, Difuntorum S, et al. In vitro activity of telavancin against resistant gram-positive bacteria. Antimicrob Agents Chemother 2008;52:2647-52.

22. Lockhart SR, Abramson MA, Beekman SE, et al. Antimicrobial resistance among gram-negative bacilli as causes of infections in intensive care unit patients in the United States between 1993 2004. J Clin Microbiol 2007;45:3352-59.

23. Landman D, Georgescu C, Martin DA, et al. Polymyxins revisited. Clin Microbiol Rev 2008;21:449-65.

24. Conly JM, Johnston BL. Colistin: The phoenix rises. Can J Infect Dis Med Microbiol 2006;17:267-69. 


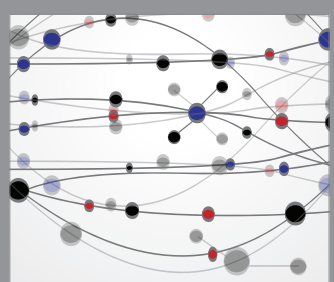

The Scientific World Journal
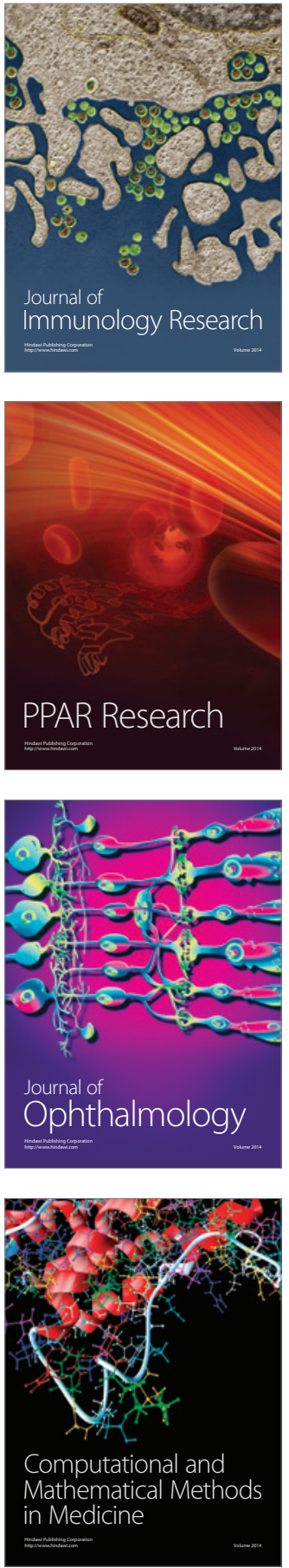

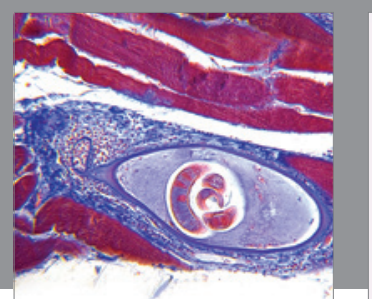

Gastroenterology Research and Practice

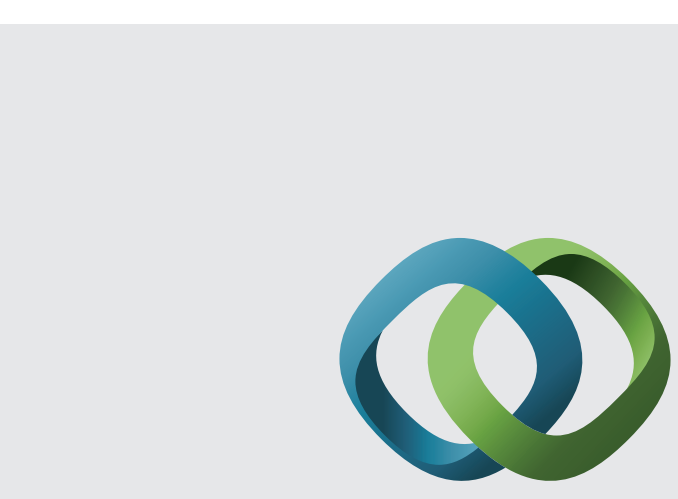

\section{Hindawi}

Submit your manuscripts at

http://www.hindawi.com
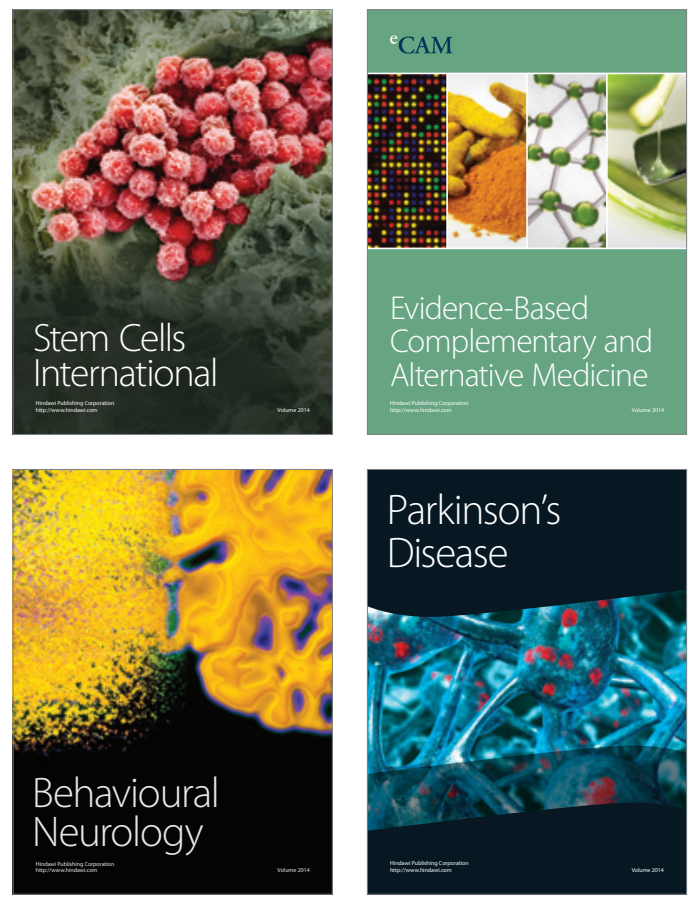
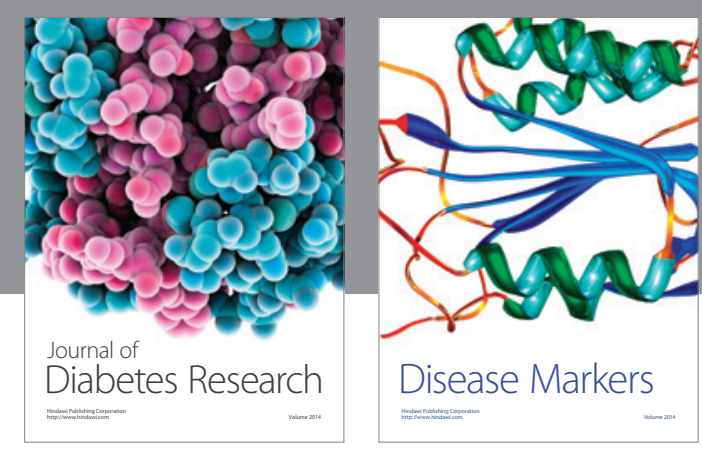

Disease Markers
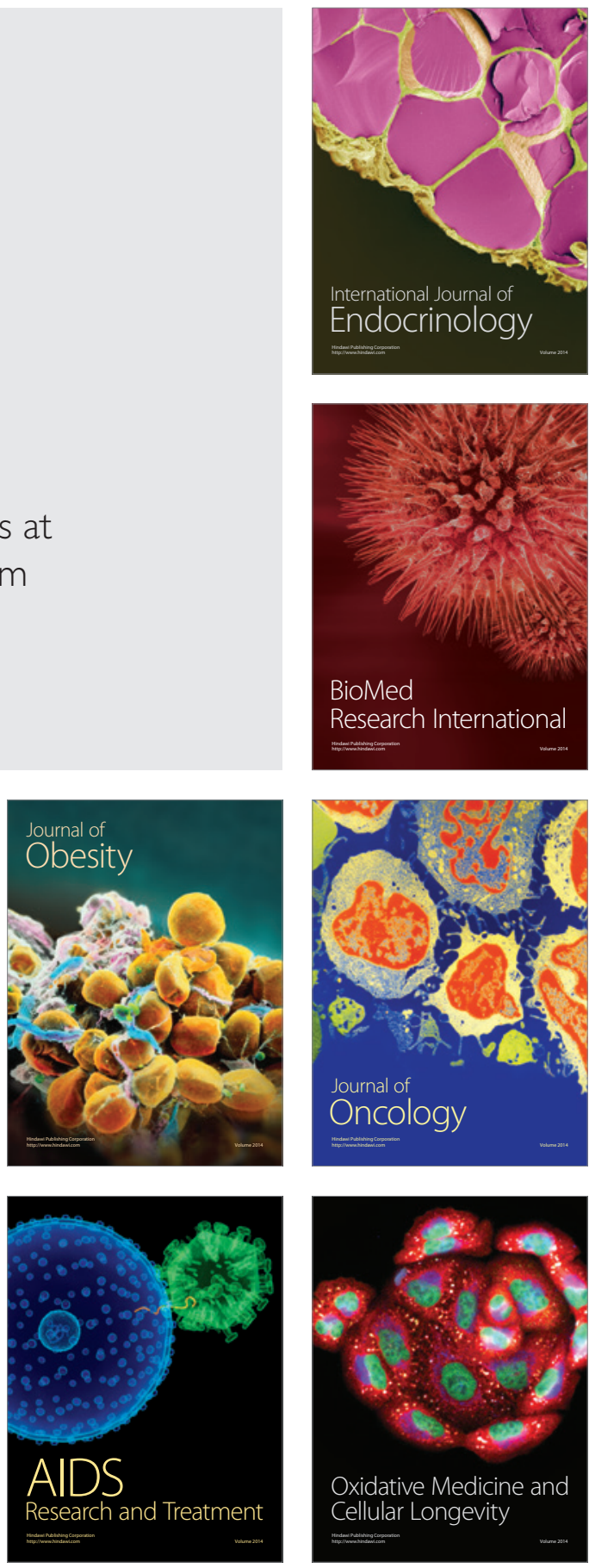